\title{
Physical properties of brightest cluster galaxies up to redshift 1.80 based on HST data ${ }^{\star}$
}

\author{
A. $\mathrm{Chu}^{1}$, F. Durret ${ }^{1}$, and I. Márquez ${ }^{2}$ \\ 1 Sorbonne Université, CNRS, UMR 7095, Institut d'Astrophysique de Paris, 98bis Bd Arago, 75014 Paris, France \\ e-mail: aline.chu@iap.fr \\ 2 Instituto de Astrofísica de Andalucía, CSIC, Glorieta de la Astronomía s/n, 18008 Granada, Spain \\ Received 26 December 2020 / Accepted 28 January 2021
}

\begin{abstract}
Context. Brightest cluster galaxies (BCGs) grow by accreting numerous smaller galaxies, and can be used as tracers of cluster formation and evolution in the cosmic web. However, there is still controversy regarding the main epoch of formation of BCGs; some authors believe they already formed before redshift $z=2$, while others find that they are still evolving at more recent epochs.

Aims. We study the physical properties of a large sample of BCGs covering a wide redshift range up to $z=1.8$ and analyzed in a homogeneous way, to see if their characteristics vary with redshift. As a first step we also present a new tool to determine for each cluster which galaxy is the BCG.

Methods. For a sample of 137 clusters with HST images in the optical and/or infrared, we analyzed the BCG properties by applying GALFIT with one or two Sérsic components. For each BCG we thus computed the Sérsic index, effective radius, major axis position angle, and surface brightness. We then searched for correlations of these quantities with redshift.

Results. We find that the BCGs follow the Kormendy relation (between the effective radius and the mean surface brightness), with a slope that remains constant with redshift, but with a variation with redshift of the ordinate at the origin. Although the trends are faint, we find that the absolute magnitudes and the effective radii tend to become respectively brighter and bigger with decreasing redshift. On the other hand, we find no significant correlation of the mean surface brightnesses or Sérsic indices with redshift. The major axes of the cluster elongations and of the BCGs agree within $30^{\circ}$ for $73 \%$ of our clusters at redshift $z \leq 0.9$.

Conclusions. Our results agree with the BCGs being mainly formed before redshift $z=2$. The alignment of the major axes of BCGs with their clusters agree with the general idea that BCGs form at the same time as clusters by accreting matter along the filaments of the cosmic web.
\end{abstract}

Key words. galaxies: clusters: general - galaxies: bulges

\section{Introduction}

Galaxy clusters are the largest and most massive gravitationally bound structures observed in the Universe. They are the perfect probes to test cosmological models and help us better understand the history of the Universe as they will constrain the limits of observed physical parameters through time, such as mass or brightness, in numerical simulations (Kravtsov \& Borgani 2012). The $\Lambda$ cold dark matter $(\Lambda C D M)$ model proposes a hierarchical evolution scenario starting from small fluctuations that assemble together via the gravitational force, and grow to form bigger and bigger structures. As a result, galaxy clusters are the latest and most massive structures to have formed.

Clusters are believed to be located at the intersection of cosmic filaments, and to form by merging with other smaller clusters or groups of galaxies, and by constantly accreting gas and galaxies that preferentially move along cosmic filaments and end up falling towards the center of the gravitational potential well, which often coincides with the peak of the X-ray emission (see De Propris et al. 2020, and references therein). Generally, the brightest galaxy in the cluster, the brightest cluster galaxy (BCG) lies at the center of the cluster. It is usually a supermas-

\footnotetext{
* Full Tables 1-4 are only available at the CDS via anonymous ftp to cdsarc.u-strasbg. fr $(130.79 .128 .5)$ or via http://cdsarc. u-strasbg.fr/viz-bin/cat/J/A+A/649/A42
}

sive elliptical galaxy that is formed and grows by mergers with other galaxies, and can be up to two magnitudes brighter than the second brightest galaxy. This property makes BCGs easily recognisable. BCGs have often been referred to as $\mathrm{CD}$ galaxies (i.e., supergiant ellipticals with a large and diffuse halo of stars). Since their properties are closely linked to those of their host cluster (Lauer et al. 2014), they can be extremely useful to trace how galaxy clusters have formed and evolved. BCGs tend to be aligned along the major axis of the cluster, which also hints at the close link between the BCG and its host cluster (Donahue et al. 2015; Durret et al. 2016; West et al. 2017; De Propris et al. 2020). This alignment suggests that the accretion of galaxies may occur along a preferential axis, with galaxies falling into clusters along cosmic filaments.

Most of the stars in today's BCGs were already formed at redshift $z \geq 2$ (Thomas et al. 2010). BCGs, especially the most massive ones, can present an extended halo made of stars that were stripped from their host galaxy during mergers, and form the intracluster light (ICL). When measuring photometric properties of galaxies, some parameters such as the BCG major axis can be difficult to measure accurately as the separation between the ICL and the external envelope of the BCG is not clear. However, the ICL is a very faint component, and as we are observing bright galaxies the ICL should not strongly affect our study, so the ICL is not considered in this paper. 
The evolution of BCG properties with redshift is of interest in the study of cluster formation and evolution, but this topic remains quite controversial. Some authors report no evolution of the sizes of the BCGs with redshift (see Bai et al. 2014; Stott et al. 2011, and references therein). Stott et al. (2011) found that there was no significant evolution of the sizes or shapes of the BCGs between redshift 0.25 and 1.3 ; instead, Ascaso et al. (2010) found that although the shapes show little change, they have grown by a factor of 2 in the last $6 \mathrm{Gyr}$. Bernardi (2009) found a 70\% increase in the sizes of BCGs since $z=0.25$, and an increase of a factor of 2 since $z=0.5$.

Bai et al. (2014) found that while the inner region of the galaxies does not grow much, the light dispersed around the BCG forms the outer component, resulting in a shallow outer luminosity profile. This is an indication of an inside-out growth of BCGs: the inner component forms first and then stops growing while the outer component develops. Edwards et al. (2019) gave more evidence to justify this inside-out growth of BCGs by showing that the stars in the ICL are younger and less metal rich than those in the cores of the BCGs. They also showed that the most extended BCGs tend to be close to the X-ray center. This last statement is supported by Lauer et al. (2014), who added that the inner component would have already been formed before the cluster, while the outer component, the envelope of the BCG, is formed and grows later. Numerical simulations with AGN suppressed cooling flows show that about $80 \%$ of the stars are already formed at redshift $z \approx 3$ in the BCG progenitors that merge together to form today's BCGs (De Lucia \& Blaizot 2007). Cooke et al. (2019a) found that BCG progenitors in the COSMOS field have an active star formation phase before $z=$ 2.25 , followed by a phase of dry and wet mergers until $z=1.25$ that leads to more star formation and increases the stellar mass of the progenitors, after which the stellar mass of progenitors mainly grows through dry mergers, and half of the stellar mass is formed at $z=0.5$. Similarly, Cerulo et al. (2019) did not find significant stellar mass growth between $z=0.35$ and $z=0.05$, suggesting that most of the BCGs stellar masses were formed by $z=0.35$. Durret et al. (2019) observed a possible variation with redshift of the effective radius of the outer Sérsic component of BCGs for 38 BCGs in the redshift range $0.2 \leq z \leq 0.9$, agreeing with a scenario in which BCGs at these redshifts mostly grow by accreting smaller galaxies.

Several conflicts also arise on the growth of the stellar masses of the BCGs. Collins \& Mann (1998), Collins et al. (2009), and Stott et al. (2010) found little to no evolution. On the other hand, other studies found a strong evolution in the stellar masses of BCGs since redshift $z=2$ (Aragon-Salamanca et al. 1998; Lidman et al. 2012; Lin et al. 2013; Bellstedt et al. 2016; Zhang et al. 2016).

In the present paper we characterize how the properties of BCGs have evolved since $z=1.80$, based on HST data to have the best possible spatial resolution, which is particularly necessary at high redshift. When dealing with a large amount of data, identifying the BCG of a cluster to build a sample can be a long task. This is why we present here a method based on several photometric properties of the BCGs that will allow us to detect BCGs automatically. We analyzed a sample of 137 galaxy clusters, covering the redshift range $0.187 \leq z \leq 1.80$ and various types of BCGs, including star forming BCGs (SF BCGs), interacting BCGs, hosts of possible AGNs, and supercluster members.

The present paper covers a large redshift range with one of the largest samples observed with HST (see Fig. 1, which is described in more detail in the next section). This will enable

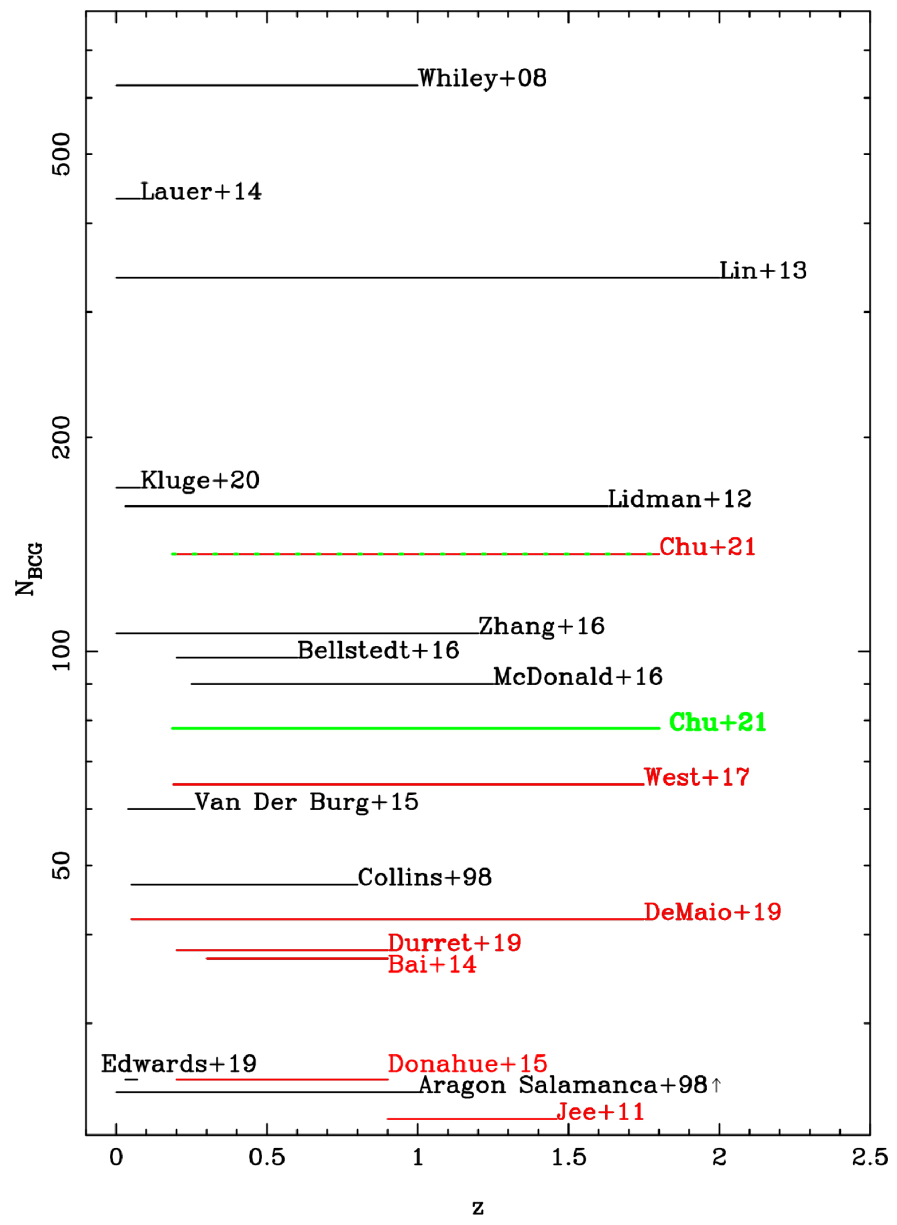

Fig. 1. Comparison of the various samples of BCGs found in the literature, considering the redshift range, the number of galaxies analyzed, and the type of data used. Only samples with at least 20 objects are represented here. Cerulo et al. (2019) with a sample of 74275 BCGs is not represented here for reasons of legibility. The samples represented in black use ground-based telescope data, space-based data excluding HST, or a mix of ground-based and space-based data, while those in red only use HST data. Our initial sample is represented by the red and green dashed line, and our final BCG sample is in green (see Sect. 4).

us to obtain more significant statistics on the evolution of BCG properties.

The paper is organized as follows. We describe the data in Sect. 2, the method to automatically detect the BCGs in Sect. 3, and the modeling of their luminosity profiles in Sect. 4. The results obtained as well as a short study of the link between the BCG masses, the distance between the BCG and the X-ray center of the cluster, and the physical properties are given in Sect. 5. A final discussion and conclusions are presented in Sect. 6.

Throughout this paper, we assume $H_{0}=70 \mathrm{~km} \mathrm{~s}^{-1} \mathrm{Mpc}^{-1}$, $\Omega_{\mathrm{M}}=0.3$, and $\Omega_{\Lambda}=0.7$. The scales and physical distances are computed using the astropy.coordinates package ${ }^{1}$. Unless specified, all magnitudes are given in the $\mathrm{AB}$ system.

\section{Sample and data}

\subsection{Sample}

The sample studied in this paper consists of 137 galaxy clusters with HST imaging taken from Jee et al. (2011),

\footnotetext{
1 https://docs.astropy.org/en/stable/coordinates/
} 


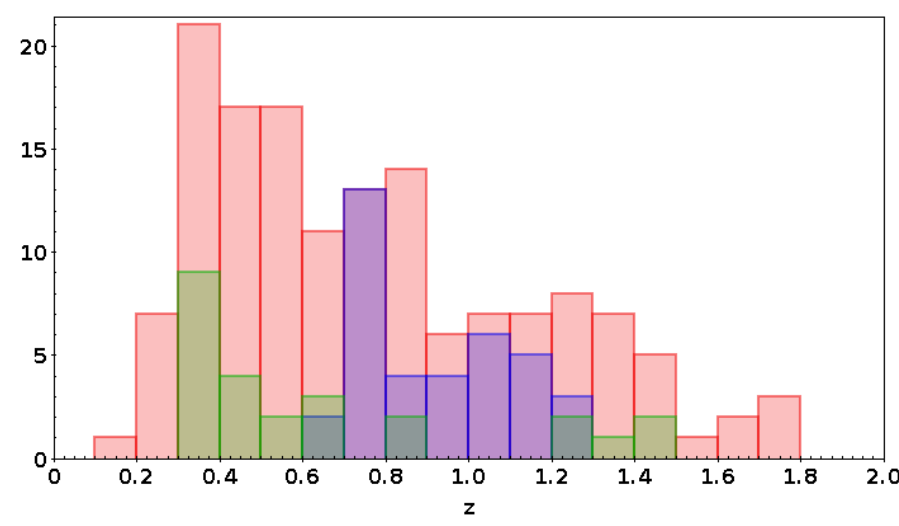

Fig. 2. Histogram of the redshifts of the 149 BCGs in our sample. The red histogram shows all the BCGs studied, while the blue histogram (37 BCGs) shows those observed in rest frame filters that are too blue compared to the $4000 \AA$ break (see Sect. 3). The green histogram (25 BCGs) shows all BCGs with an important inner component (see Sect. 4).

Postman et al. (2012a), Bai et al. (2014), Donahue et al. (2015), West et al. (2017), DeMaio et al. (2019), Durret et al. (2019), and Sazonova et al. (2020). We also add five more distant clusters at $z \geq 0.8$, as well as the cluster Abell 2813 at $z=0.29$. Among them, we identify 12 clusters that have in their center two BCGs similar in magnitude and size (see Sect. 3). As a result, our final BCG sample contains 149 BCGs; the number of BCGs is not equal to the number of clusters studied because of the clusters with two BCGs. This sample is a good representation of the most massive BCGs in the range $0.1 \leq z \leq 1.80$. The redshift distribution is shown in Fig. 2.

All of these clusters have data available from the Hubble Space Telescope, obtained with the Advanced Camera for Surveys (ACS) in optical bands and/or the Wide Field Camera 3 (WFC3) in infrared bands, resulting in good-quality images. This allows us to perform accurate photometry with relatively good precision, and to treat all the BCGs in a homogeneous way. Contrary to other studies such as Bai et al. (2014), we do not exclude from our study clusters with bright nearby objects that may hinder our measurements near the BCG area. We also identify in our sample two clusters that host blue BCGs (negative rest frame blue-red color), with active star forming regions inside the BCGs. These two BCGs will be described in more detail in Sect. 3.

Our sample covers a large redshift range, which will enable us to trace the history of cluster formation through time. Figure 1 shows the comparison of the sample sizes and redshift ranges between different studies done on BCGs ${ }^{2}$. Cerulo et al. (2019) studied a sample 74275 BCGs from the SDSS in the redshift range $0.05 \leq z \leq 0.35$, and is not represented in this figure for better legibility. Most large studies, especially those with HST data (represented in red), were done exclusively on local BCGs $(z \leq 0.1)$ (Lauer et al. 2014; Cerulo et al. 2019), while farther clusters and BCGs were limited to relatively small samples $(N \leq 45)$ (Bai et al. 2014; DeMaio et al. 2019; Durret et al. 2019) and/or used ground-based data (represented in black). Our sample contains more clusters and BCGs at high redshifts $(z \geq$ 0.7) than that of Lidman et al. (2012) (73 and 33, respectively).

2 By increasing number of BCGs: Bai et al. (2014), Durret et al. (2019), DeMaio et al. (2019), van der Burg et al. (2015), West et al. (2017), McDonald et al. (2016), Bellstedt et al. (2016), Zhang et al. (2016), Lidman et al. (2012), Kluge et al. (2020), Lin et al. (2013), Lauer et al. (2014), Whiley et al. (2008), and Cerulo et al. (2019).
With the present study, we therefore almost double the previous samples and cover a larger range in redshift. This enables us to obtain more significant statistics on the evolution of BCG properties.

Lidman et al. (2012), Lin et al. (2013), West et al. (2017), and De Propris et al. (2020) mainly focus on the alignment of BCGs with their host cluster and on the evolution of the BCG stellar masses. Our work constitutes a deeper analysis since we also study the luminosity profiles of the BCGs.

\subsection{Retrieving data and cluster information}

We retrieved all the FITS images from the Hubble Legacy Archive $\left(\mathrm{HLA}^{3}\right)$. We looked for combined or mosaic images according to what is available, and downloaded stacked images directly from the HLA. To avoid handling such heavy files, we first cropped these images, defined the new center on the cluster coordinates found in NED, and created a new image that was $1.2 \mathrm{Mpc}$ wide. Linear scales $\left(\operatorname{arcsec} \mathrm{Mpc}^{-1}\right)$ were determined from the cluster redshifts in the literature (from Jee et al. 2011; Postman et al. 2012a; Bai et al. 2014; Donahue et al. 2015; West et al. 2017; DeMaio et al. 2019; Durret et al. 2019; Sazonova et al. 2020, or were found in NED for the five other clusters we added). Cluster information can be found in Table 1. It was necessary to add the keywords "GAIN" and "RDNOISE" in the header of the FITS images, to be used later by SExtractor or GALFIT. As the images are in units of electrons $\mathrm{s}^{-1}$, we set the GAIN to 1 and multiplied the images by the total exposure time (EXPTIME) to get back to units in electrons. Single exposure images were summed with AstroDrizzle to get the final combined images. We also retrieved the associated weight maps (wht fits) obtained applying the inverse variance map (IVM) option of AstroDrizzle.

\section{Procedure for the detection of the BCG}

The definition of the BCG that we use throughout this paper is the following. The BCG is the brightest galaxy in the cluster that lies close to the cluster center, defined as the center of the cluster member galaxy distribution. Generally, the cluster center is defined as the X-ray center of the cluster as X-rays trace the mass distribution better. However, it is difficult to obtain X-ray data, particularly at high redshifts. If a large sample is considered, most probably a good fraction of the clusters do not have X-ray data available. Moreover, it has been shown in several studies (Patel et al. 2006; Hashimoto et al. 2014; De Propris et al. 2020) that BCGs are often displaced from the X-ray center. For these reasons, and anticipating future works with much larger samples, we use a definition that is independent from X-rays and only relies on optical and infrared photometric data. We define the center as that of the spatial distribution of cluster galaxies (as in Kluge et al. 2020). X-ray coordinates are only available for 68 out of the 137 clusters in our sample (see Table 4). These X-ray positions are only used to study whether or not the BCG properties correlate with their position relative to the X-ray center (see Sect. 5).

\subsection{Method for detecting red BCGs}

The method applied to automatically select red BCG is schematically summarized in Fig. 3, and is described in detail below. The

\footnotetext{
https://hla.stsci.edu/
} 
Table 1. Sample of the 149 BCGs studied in this paper.

\begin{tabular}{|c|c|c|c|c|c|c|c|c|}
\hline Name & $\begin{array}{l}\mathrm{RA}_{\mathrm{BCG}} \\
(\mathrm{J} 2000)\end{array}$ & $\begin{array}{l}\operatorname{Dec}_{B C G} \\
(\mathrm{~J} 2000)\end{array}$ & Redshift & Class & Instrument & Filter & $\begin{array}{l}\text { Scale } \\
\left(\mathrm{kpc} /{ }^{\prime \prime}\right)\end{array}$ & Color \\
\hline SPT-CLJ000C & 2504 & 57.8093 & 2 & 1 & ACS_WFC & $F 81$ & 7.128 & $F 606$ \\
\hline $\mathrm{Cl} 0016+1609$ & 4.64 & 16.4378 & 55 & 1 & ACS_WFC & $F 850 L P$ & 2. & $F 606 W$ \\
\hline SpARCS-J0335 & & -43.2065 & & 1 & WFC̄3_IR & $F 140 W$ & & $F 105 W-F 140 W$ \\
\hline $\mathrm{ACO} 2813$ & .8528 & -20.6282 & 0.2924 & 1 & ACS_WFC & $F 814 W$ & 4.368 & $F 435 W-F 606 W$ \\
\hline $\mathrm{ACO} 2813$ & 10.8548 & -20.6169 & 0.2924 & 2 & ACS_WFC & $F 814 W$ & 4.368 & $F 435 W-F 606 W$ \\
\hline XDCPJ0044-2033 & 11.0236 & -20.5651 & 1.59 & 1 & WFC3_IR & $F 160 W$ & 8.42 & $F 105 W-F 140 W$ \\
\hline RXJ0056-27 & 14.2374 & -27.675 & 0.56 & 1 & ACS_W̄'WC & $F 814 W$ & 6.449 & \\
\hline SPT-CLJ0102-4915 & 15.721 & -49.2528 & 0.87 & 1 & WFC3_IR & $F 105 W$ & 7.681 & $F 625 W-F 775 W$ \\
\hline SPT-CLJ0102-4915 & 15.7409 & -49.2719 & 0.87 & 2 & ACS_WFC & $F 850 L P$ & 7.681 & $F 625 W-F 775 W$ \\
\hline RXJ0110+19 & 17.5758 & 19.6387 & 0.317 & 1 & ACS_WF-WC & $F 814 W$ & 4.617 & \\
\hline$\ldots$ & $\ldots$ & $\ldots$ & $\ldots$ & $\ldots$ & $\ldots$ & $\ldots$ & $\ldots$ & $\ldots$ \\
\hline
\end{tabular}

Notes. The columns are: full cluster name, coordinates of the BCG, redshift, class of the BCG (if two BCGs are defined for a cluster, class 1 represents the brighter of the two), instrument, filter used to model the luminosity profile of the BCG (see Sect. 4), associated scale, color computed to extract the red sequence of the cluster (see Sect. 3). The BCGs with no values in the last column only had data in one filter, and their coordinates were taken from the literature. The full table is available at the CDS.

efficiency of the method is discussed in Sect. 3.1.4. Blue BCGs are mentioned in Sect. 3.2.

\subsubsection{Rejection of foreground sources}

In order to differentiate the BCG from other objects in the field, we need to identify which objects are part of the cluster and which are not. We describe below our method for detecting the BCGs among all the contaminations (stars, foreground and background galaxies, artifacts) in our images.

Measurements with SExtractor (see Bertin \& Arnouts 1996, for more details on the parameters) were done using two different deblendings (parameters DEBLEND_MINCOUNT $=0.01$ and DEBLEND_MINCOUNT=0.02). The smallest deblending parameter (i.e., the finest deblending) is sufficient to separate two nearby galaxies without fragmenting excessively spiral galaxies in the foreground, and provides the most accurate measurements. However, BCGs can present a very diffuse and luminous halo which may be associated with ICL. We found that, in the presence of nearby bright sources in the region of the BCG, SExtractor would detect only those foreground sources and process the BCG halo as a very luminous background. We therefore decided to run SExtractor in parallel with a coarser deblending to take this into account. The two catalogs obtained with two different deblending parameters are then matched; we keep the values obtained with the finer deblending, and add all new objects detected using a coarser deblending.

We computed the magnitude at which our catalog is complete at $80 \%, m_{80 \%}$. To achieve this we plot the histogram in apparent magnitudes and fit the distribution up to the magnitude at which the distribution drops. By dividing the number of detected sources by the total number of sources expected to be detected in a magnitude bin (given by the fit), we compute the completeness of the catalog at each bin. We can then determine $m_{80 \%}$, and make a cut in apparent magnitude to reject all galaxies with $m \geq m_{80 \%}+2$, as the photometry would not be accurate for these faintest objects.

Our procedure to reject the various contaminations is as follows. First, we query in NED for all the sources in the region of the cluster and reject all those with a spectroscopic redshift that differs by more than 0.15 from the cluster redshift $\left(\left|z-z_{\text {spec }}\right|>0.15\right)$. We identify bad detections by their mag- nitude values, which get returned as MAG $=99.99$ by SExtractor. All point sources or unresolved compact galaxies are eliminated using the parameter CLASS_STAR $\geq 0.95$ in SExtractor, which requires a point spread function (PSF) model to be fed into SExtractor, created with PSFex (Bertin 2011). Most foreground galaxies can be identified by their excessively bright absolute magnitude when computed from their MAG_AUTO magnitude and assuming they are at the cluster redshift. We thus exclude all sources with an absolute magnitude MAG_ABS $\leq-26$.

We can identify edge-on spiral galaxies, which appear very elongated. They can be filtered by making cuts in elongation (defined in SExtractor as the ratio of the galaxy's major to minor axis). As we explain in Sect. 3.1.2, we consider two different filters. We define two different cuts depending on the filter we are looking at: in the bluest filter we apply the criterion ELONGA$\mathrm{TION} \leq 2.3$, and in the reddest filter ELONGATION $\leq 2.6$. The latter limit may seem quite high to filter efficiently all edge-on spiral galaxies. However, because of deblending issues, measuring with precision the lengths of the major and minor axes of the sources can be difficult, and will sometimes lead to a very elongated object. A very bright and elongated halo around the BCG, which can be linked to the ICL, will possibly return a high $a / b$ axis ratio. This is the case for the BCG in RX J2129+0005, which has the highest $a / b$ elongation (in the $F 606 W$ filter) measured in our sample, reaching $a / b=2.57$ (see Fig. 4). As the reddest filter is more sensitive to the ICL, we prefer to define a limit that is not too strict on this filter. It does not eliminate all edge-on galaxies (which would need a lower limit), but we cannot take the risk of filtering out any of the BCGs we are looking for. This is the reason why we define a different stricter limit on the bluest filter, as it is more sensitive to the blue stellar population present in the disk of spiral galaxies, and less to the ICL.

\subsubsection{Selection of red cluster galaxies}

Early-type galaxies in clusters are usually easily recognizable by their red color, since they are mostly red elliptical galaxies, without star formation. While blue spiral galaxies also exist inside the cluster, they are a minority, and red elliptical galaxies draw a red sequence in a color-magnitude diagram, which has a low dispersion. We thus apply a filter in color in order to only keep the red galaxies that form the red sequence. 


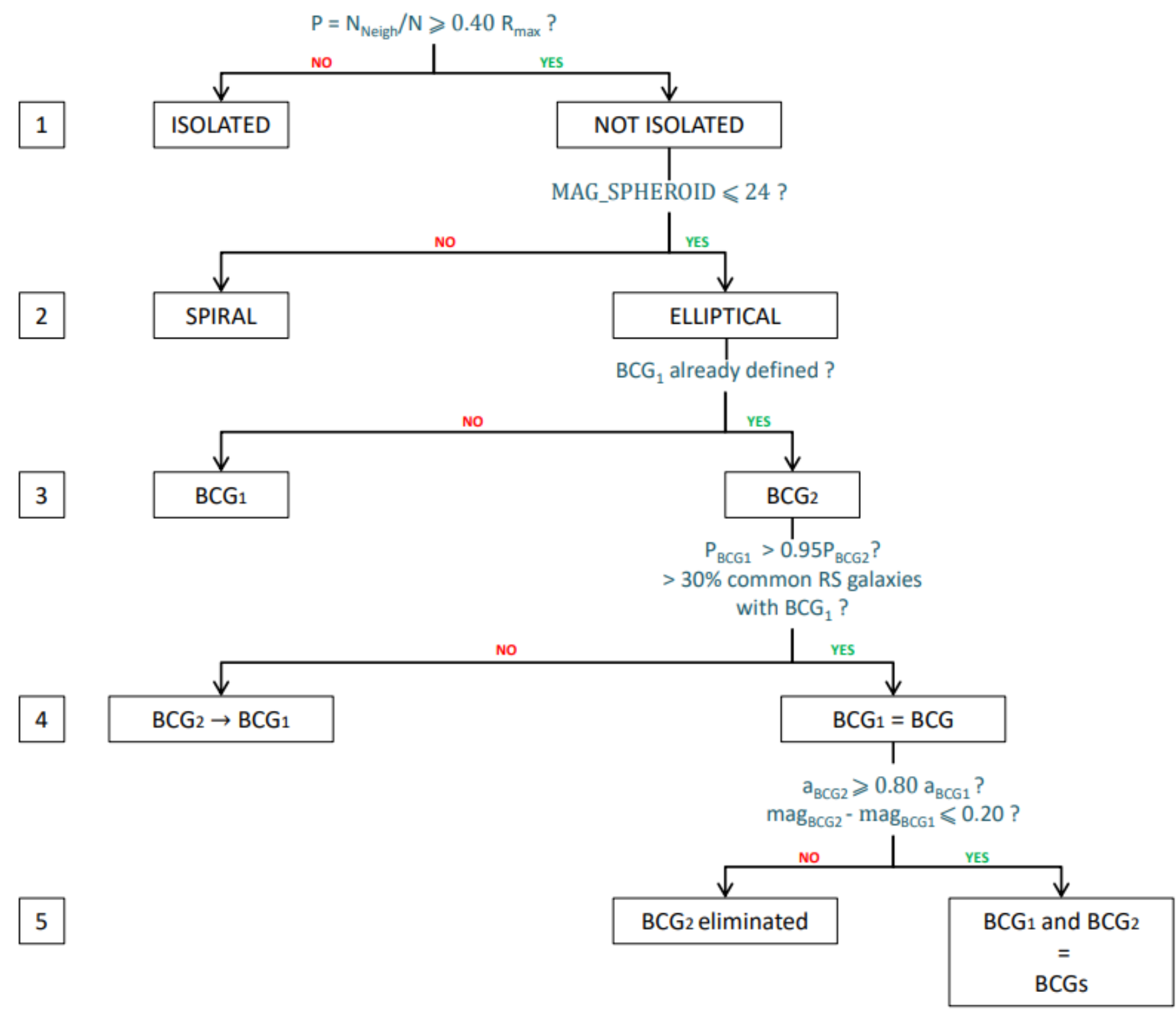

Fig. 3. Flowchart showing how BCGs are automatically selected, in the case of a rich cluster (see description of the method for our definition of a rich cluster). Values may change for less rich clusters (see Sect. 3.1.3).

To extract all the red early-type galaxies in a cluster at redshift $z$, we model their color using a spectral energy distribution (SED) template from Bruzual \& Charlot (2003). The model is similar to the one used by Hennig et al. (2017): a single period of star formation beginning at redshift $z_{\mathrm{f}}=5$, with a Chabrier IMF and solar metallicity, that decreases exponentially with $\tau=0.5$ Gyr. However, it differs from the Hennig et al. (2017) model on the star formation redshift; we chose a higher $z_{\mathrm{f}}$ to better model clusters at higher redshifts, while Hennig et al. (2017) limit their study to redshift $z=1.1$. We reject all blue galaxies (blue-red $\leq 0$ ) and all galaxies whose measured (bluered) color differs by more than $0.60 \mathrm{mag}$ from the model. While the red sequence of a cluster presents a rather narrow colormagnitude relation, and therefore very little dispersion, this large limit of 0.60 was fixed in order to take into account photometric uncertainties due to deblending issues, redshift uncertainties, or simply to the accuracy of the model used (Charlot, priv. comm.). The color is computed considering a fixed aperture of $35 \mathrm{kpc}$ in diameter (parameter MAG_APER), which is large enough to contain all of the galaxy's light. All magnitudes are K-corrected (K-correction values from the EZGAL BC03 computed model), and we also take into account galactic extinction. Dust maps were taken from Schlegel et al. (1998), and reddening values for the ACS and WFC3 bandpasses were taken from Schlafly \& Finkbeiner (2011), considering a reddening law $R_{V}=3.1$. The colors computed depend on the filters available and on the redshift of the cluster. The color computed for each cluster can be found in Table 1 .

The rest frame (blue-red) color to compute is defined as the color based on two magnitudes with the smallest wavelength difference that bracket the $4000 \AA$ break at the cluster redshift. Depending on what filters are available for each cluster, the selected filters will differ. An example is given in Fig. 5 for a cluster at $z=1.322$; in this case the filters bracketing the $4000 \AA$ break are $F 814 W$ and $F 105 W^{4}$. In the cases where the two optimal filters are not available, the color used to trace the red sequence galaxies at different redshifts may not be

4 The colors $F 606 W-F 625 W$ and $F 775 W-F 814 W$ were excluded as the two filters are very close to each other. 


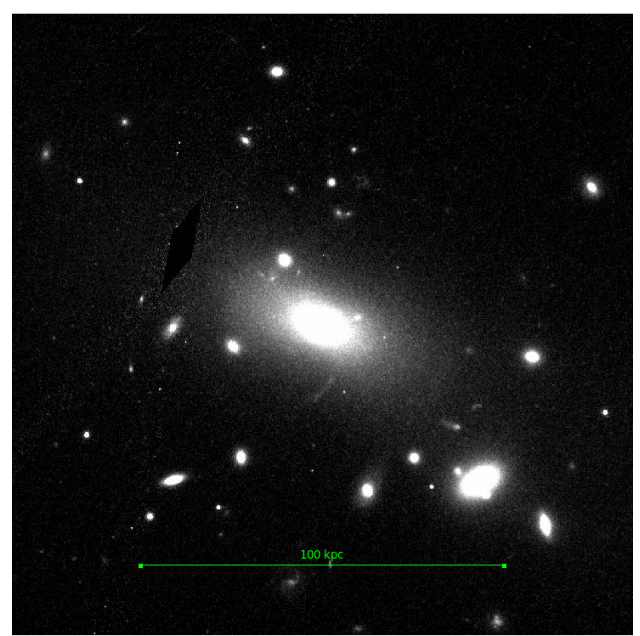

Fig. 4. BCG in RXJ2129+0005 at redshift $z=0.234$. The extended and luminous halo makes it difficult to accurately estimate the $a / b$ axis ratio. In this case the major axis has most likely been overestimated, as the diffuse light is extended along this axis. The image was taken with the $F 775 W$ ACS filter.

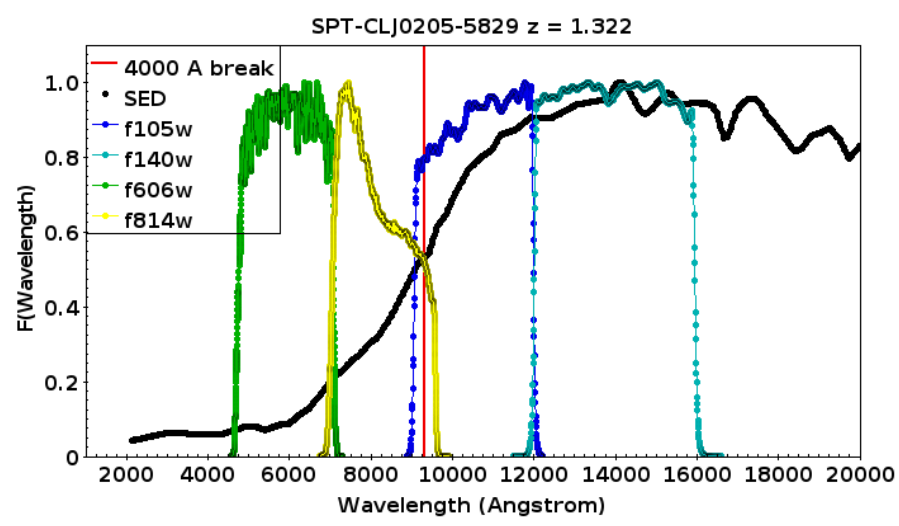

Fig. 5. SED of an elliptical galaxy from the CFHTLS (black solid line) redshifted at the cluster's redshift (SPT-CL J0295-5829, $z=1.322$ ). The filter transmissions are normalized to 1 for better visualization, and the break at $4000 \AA$ is shown as a red vertical line for reference. In this case the chosen (blue-red) rest frame color is $F 814 W-F 105 W$, and the filter chosen for the final step (modeling the luminosity profile with GALFIT) is $F 140 W$ (see Sect. 4).

efficient; for instance, the use of the color $(F 606 W-F 140 W)$ would not optimize the selection as a galaxy at higher redshift $(z=1.65$ for example) than the cluster redshift (here $z=1.322$ ) would have the same color and would not be filtered out.

\subsubsection{Rejection of spiral and isolated galaxies}

The cut in colors is an important step that allows us to remove most of the spiral galaxies and to maximize the number of ellipticals in our catalogs. However, a few foreground galaxies may still remain, and we describe here the method used to remove them.

The algorithm described hereafter is applied to every single galaxy, from the brightest to the faintest, until the cluster BCG is found. We refer the reader to the sketch shown in Fig. 3. The procedure includes the following steps:

- Step 1: For each cluster we sort the catalog from the brightest to the faintest galaxies, and going down their brightnesses, we

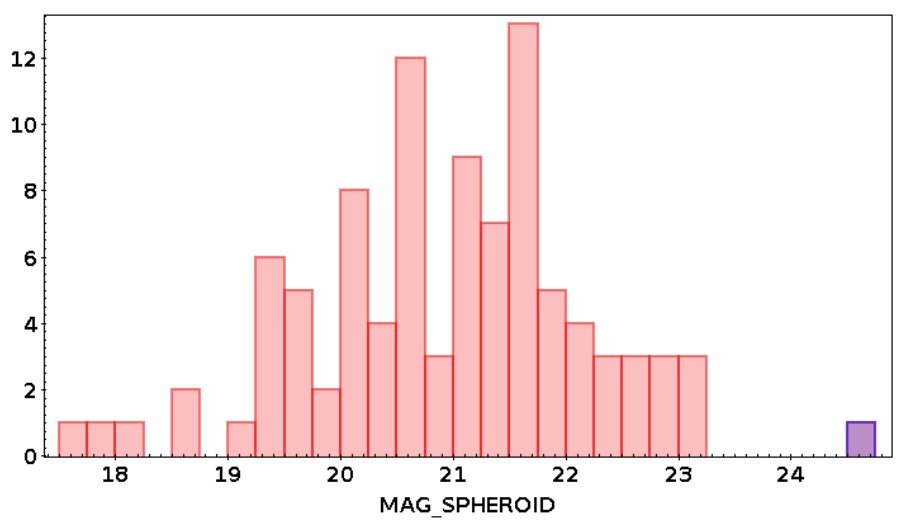

Fig. 6. Histogram of the modeled bulge magnitudes (parameter MAG_SPHEROID) returned by SExtractor. All BCGs are shown in red; the blue bin represents a spiral galaxy close to the cluster ClG J1604+4304 (see Step 2).

exclude galaxies that are too isolated from the rest. We define the BCG as the brightest elliptical galaxy at the center of the galaxy density distribution. To calculate the center we compute $N_{\text {Neigh }}$, the number of cluster members (i.e., red sequence galaxies) found in a fixed aperture of $200 \mathrm{kpc}$ radius centered on each galaxy in the final catalog, and note $N_{\max }$ the maximum number computed. If $N$ is the total number of red sequence galaxies whose colors fall within $0.60 \mathrm{mag}$ from the model, and $N_{\text {Neigh }}$ is the number of neighbors of a given galaxy in an aperture of $200 \mathrm{kpc}$, we consider that a galaxy is isolated and unlikely to be the BCG if the ratio $P=N_{\text {Neigh }} / N$ is lower than $40 \%$ of $P_{\text {max }}=N_{\max } / N$.

Considering that we cropped our images to cover a projected area of $1.2 \times 1.2 \mathrm{Mpc}^{2}$, the aperture of diameter $400 \mathrm{kpc}$ taken here represents one-third of the side of the images. This is small enough to detect high-density areas on the image, and big enough to work on clusters with a high spatial extent. After several trials adopting different values, the value of $200 \mathrm{kpc}$ radius is the one that works best. Less than $200 \mathrm{kpc}$ becomes too small for extended clusters, while a larger radius makes it difficult to detect the smaller density fluctuations, as the covered area becomes large. The limit defined at $R_{\lim }=0.40 R_{\max }$ was also determined after several tests. This condition takes into account the cluster richness and spatial extent, as well as the possible offset of the $\mathrm{BCG}$ relatively to the cluster center.

- Step 2: The next step consists in filtering out the last spiral galaxies that remain among the potential BCG candidates. We run SExtractor to model the potential BCG with a bulge and a disk component. We find that spiral galaxies have a very faint bulge (parameter MAG_SPHEROID); as can be seen in Fig. 6, a spiral galaxy (shown in blue) near the cluster ClG J1604+4304 prevented us from successfully detecting the BCG. We see a gap in magnitude between the spiral galaxy and the other BCGs (which are not all pure ellipticals). This enables us to define a new cut in magnitude to remove these remaining spirals: MAG_SPHEROID $\leq 24$.

- Step 3: If a galaxy complies with these conditions (i.e., not being isolated and not being a spiral), we keep it as $\mathrm{BCG}_{1}$ if no other $\mathrm{BCG}$ candidate was found before, and as $\mathrm{BCG}_{2}$ otherwise. We do not proceed to the next step until a $\mathrm{BCG}_{2}$ is defined.

- Step 4: We check if there are more red sequence members in the same aperture for $\mathrm{BCG}_{2}$ than the number defined in Step 1 for $\mathrm{BCG}_{1}$ by comparing their $P_{\text {Neigh }}$ ratios (defined in Step 1). We denote them $P_{\mathrm{BCG} 1}$ and $P_{\mathrm{BCG} 2}$. If $P_{\mathrm{BCG} 1} \leq 0.95 P_{\mathrm{BCG} 2}$, and if 
less than $30 \%$ of $N_{\mathrm{Neigh}, \mathrm{BCG} 2}$ are in common with $\mathrm{BCG}_{1}, \mathrm{BCG}_{1}$ is eliminated and we define $\mathrm{BCG}_{2}$ as the new $\mathrm{BCG}_{1}$.

The factor of $95 \%$ ensures that the overdensity in which $\mathrm{BCG}_{2}$ lies is significantly richer than the one in which $\mathrm{BCG}_{1}$ is. The second criterion on the number of galaxies common to $\mathrm{BCG}_{1}$ and $\mathrm{BCG}_{2}$ is to make sure that we are not replacing a BCG that is not at the very center of the cluster by another galaxy that is closer. This criterion is necessary to avoid eliminating BCGs that are a little offset from the center of the cluster where the density is higher. It allows us to check that the two galaxies are not in the same area in the sky; in other words, we check that $\mathrm{BCG}_{2}$ does not belong to the same clump (overdensity) as $\mathrm{BCG}_{1}$, or that the two galaxies do not belong to the same cluster.

- Step 5: This step is taken only if $\mathrm{BCG}_{2}$ is defined, otherwise we repeat the previous steps until it is found. If $\mathrm{BCG}_{1}$ and $\mathrm{BCG}_{2}$ are similar in size (ratio of the major axes $a_{\mathrm{BCG} 2} / a_{\mathrm{BCG} 1} \geq 0.80$ ) and brightness (magnitude difference $\operatorname{mag}_{\mathrm{BCG} 2}-\operatorname{mag}_{\mathrm{BCG} 1} \leq 0.2$ ), we keep both $\mathrm{BCG}_{1}$ and $\mathrm{BCG}_{2}$ as the $\mathrm{BCG}$ of the cluster. Otherwise, $\mathrm{BCG}_{2}$ is eliminated and $\mathrm{BCG}_{1}$ is defined as the BCG.

The values above do not always work for poor clusters (i.e., when the number of cluster members is low or when the density of red sequence galaxies is low). There is no problem when all the cluster members are concentrated in the same area (with a size comparable to the previously defined aperture); however, if the members are dispersed over the sky and cover a large area, an aperture of $200 \mathrm{kpc}$ radius becomes too small to detect density fluctuations on the sky. We thus differentiate these clusters by their number of red sequence members, $N$, and by the previously defined parameter $P_{\max }$ (see Step 1). We separate the poor clusters with $P_{\max } \leq 0.25$ and $N \leq 100$ (very extended clusters with no important density clumps), and $P_{\max } \leq 0.5$ and $N \leq 40$ (low number of red sequence galaxies, extended spatial distribution). We were not able to correctly determine the BCGs for these clusters by defining a $200 \mathrm{kpc}$ radius aperture, so for these poorer clusters, we consider a bigger aperture of $500 \mathrm{kpc}$ radius. To take into account the bigger aperture, we also modify the second criterion in Step 4. We check that the two BCGs candidates, $\mathrm{BCG}_{1}$ and $\mathrm{BCG}_{2}$, have less than $50 \%$ galaxies in common in the same aperture to guarantee that they are not both residing in the same cluster.

\subsubsection{Results for detected red BCGs}

Among the 137 clusters in our sample, 50 clusters only had one filter available, and were thus excluded from this procedure. For these 50 clusters without available colors, we visually checked the images to determine the BCG, and checked with X-ray maps or other studies before adding them to the final sample.

In order to assess the efficiency and accuracy of our detection method, we checked each detection visually and compared it with other studies and with any X-ray map we could find. We compared the X-ray map to the position of the detected BCG to make sure that it is not too far from the X-ray peak (but not necessarily located at the peak, in a radius of about $200 \mathrm{kpc}$ ).

During this verification, we found that our detection differs from that of Durret et al. (2019) and Bai et al. (2014) for the BCGs in MACS-J0717.5+3745 and SpARCS-J0224, respectively. MACS-J0717.5+3745 presents a very complex structure because is it undergoing multiple mergers (see Limousin et al. 2016; Ellien et al. 2019, and references therein). Durret et al. (2019) define the BCG as the one in the southern structure; instead, we detected a brighter galaxy in the northern structure, which we define as the BCG. We decided to keep our detection as it lies near the X-ray peak in the northern structure and is surrounded by galaxies at the cluster's redshift. We found, by checking visually, that the BCG in SpARCS-J0224 defined in Bai et al. (2014) is a spiral galaxy. We thus choose to keep our detection, which is an elliptical galaxy located just south of their detection.

A few star forming BCGs can be found in our sample. We find red BCGs with a very high star formation rate (SFR), for example MACS J0329.6-0211 at $z=0.45$. This BCG has an almost starburst level of UV continuum and star formation (Donahue et al. 2015). Images of this BCG in the UV continuum and $\mathrm{H} \alpha-[\mathrm{NII}]$ lines are given by Fogarty et al. (2015), illustrating the distribution of star formation throughout the galaxy. The high SFR of about $40 M_{\odot} \mathrm{yr}^{-1}$ was confirmed by Fogarty et al. (2017), based on Herschel data. Green et al. (2016) also indicate that this galaxy hosts an AGN, and is quite blue (bluered $=-0.71$, with strong emission lines and a rather high X-ray luminosity of $11.85 \times 10^{44} \mathrm{erg} \mathrm{s}^{-1}$.

Overall, all the red BCGs, even those that are not pure elliptical BCGs or those with colors that are not optimized because of the lack of available filters, were successfully detected with our method. We successfully detect $97 \%$ of the BCGs in our sample, and all the red BCGs are found. The method is effective in detecting red BCGs presenting different morphologies and characteristics (mergers, star forming, traces of dust in the core, disturbed).

It should be noted, however, that this method may be less reliable for poorer clusters (as defined in the previous subsection). As we were conducting several tests, trying different values of apertures in which we computed the number of red sequence galaxies or the threshold below which galaxies are considered isolated, we found that the detection efficiency for poorer clusters was more sensitive to these parameters. As this method relies on the density of red sequence galaxies in a small aperture, BCGs that are a little offset from the density peak (which is more difficult to calculate for poor clusters with an extended spatial distribution) could be eliminated, and rejected as being isolated from the other red sequence galaxies.

It is also important to note that, in the presence of more than one cluster (i.e., two clusters interacting with each other) or in the case of superclusters, only the brightest galaxy of one substructure will be detected. For MACS-J0717.5+3745 for example, the BCG of the northern clump is the brightest, and thus is the one detected by our algorithm.

\subsection{Finding blue BCGs}

Out of the 98 BCGs ( 87 clusters, 11 clusters with two BCGs) in our sample that we tried to detect, we find two peculiar BCGs with blue colors. Most brightest cluster galaxies are quiescent galaxies, and their dominant stellar population is typically red and old. As they grow by undergoing mergers through time, all their gas is consumed, and we expect the star formation to be quenched or suppressed. However, we do observe, both today and in the distant universe, BCGs with intense UV emitting filaments or knots, hinting at active star formation. Cerulo et al. (2019) found that $9 \%$ of their sample of massive BCGs in the redshift range $0.05 \leq z \leq 0.35$ from the SDSS and WISE surveys have blue colors (which they define as galaxies with colors $2 \sigma$ bluer than the median color of the cluster red sequence), and are star forming. What we refer to from now on as star forming BCGs (SF BCGs), to date have only been observed in cool core clusters. Their morphology can be quite different from that of a simple elliptical galaxy, as was stated before. These galaxies can 

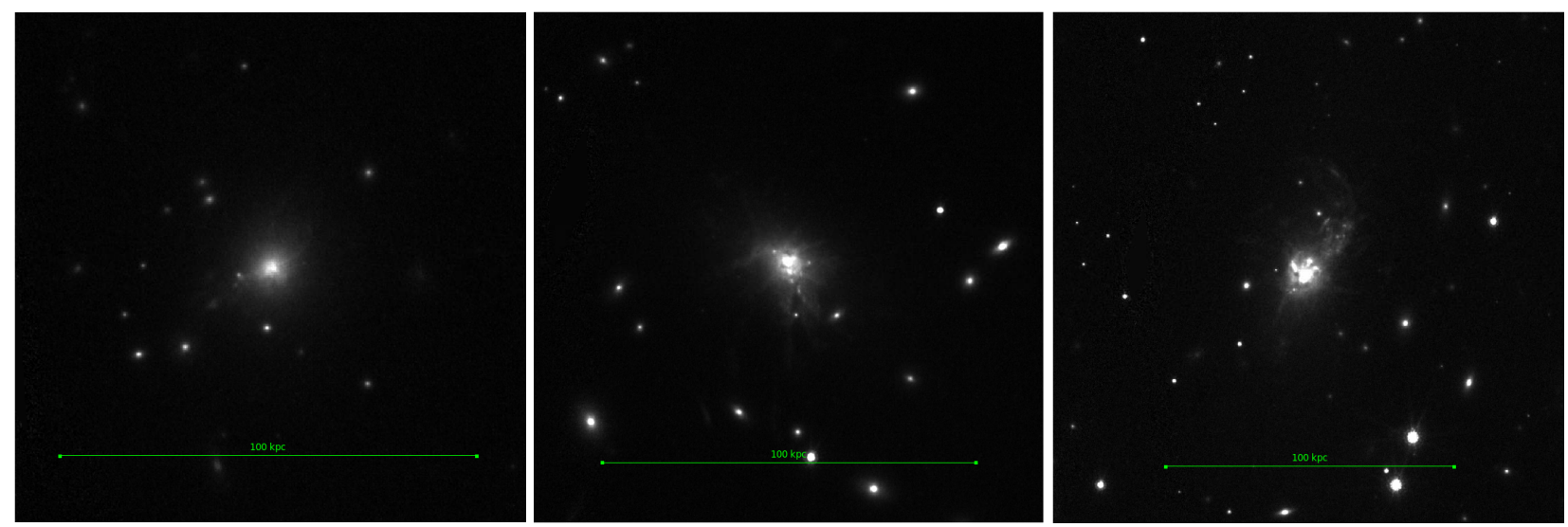

Fig. 7. From left to right: MACS J0329-0211 $(z=0.45)$, an example of a red star forming BCG; RX J1532+3020 $(z=0.3615)$ and MACS J1932$2635(z=0.352)$, the only two blue star forming BCGs in our sample.

appear disturbed, with a complex structure showing a possible recent or ongoing merger. Such examples of SF BCGs show that BCGs are not all simple ellipticals.

Two BCGs, RXJ1532+3020 $(z=0.3615)$ and MACS $\mathrm{J} 1932-2635(z=0.352)$, were not correctly detected as they are cool core BCGs with an extremely active star forming center, so they were eliminated because of their blue colors. These two BCGs were identified by eye and added manually, after checking and confirming with other studies. Their images are shown in Fig. 7.

These two BCGs are the only blue BCGs in our sample (blue meaning a negative rest frame blue-red color) out of the 98 BCGs for which we compute a color. In comparison with other studies, we find a few other BCGs that are star forming but still red.

$\mathrm{RX} \mathrm{J1532+3020}$ is one of the most extreme cool core galaxy clusters observed today, as well as one of the most massive. An intensive study by Hlavacek-Larrondo et al. (2013) shows the existence of a western and an eastern cavity, which are used to quantify the AGN feedback at the center of the galaxy. These authors estimated that this feedback would release at least $10^{45} \mathrm{erg} \mathrm{s}^{-1}$, which would prevent the intracluster medium (ICM) from cooling, and would then allow us to solve the cooling flow problem in cool core clusters. The BCG of this cluster is a radio loud galaxy that, in its central regions, presents UV filaments and knots, as well as traces of dust, hinting at recent star formation, with a SFR of at least $100 M_{\odot} \mathrm{yr}^{-1}$ (Castignani et al. 2020). A strong and broad Lyman- $\alpha$ emission and stellar UV continuum, and no other emission lines, have been observed by Donahue et al. (2016). CO with a large reservoir of molecular gas and with a high level of excitation have also been detected by Castignani et al. (2020).

MACS J1932-2635 is another cool core cluster with a huge reservoir of cold gas in the core, of mass $(1.9 \pm 0.3) \times 10^{10} M_{\odot}$, which makes it one of the largest reservoirs observed today, in which Fogarty et al. (2019) detected CO emission as well as UV knots and $\mathrm{H} \alpha$ filaments around the BCG. They measured a SFR of $250 M_{\odot} \mathrm{yr}^{-1}$, and also observed an elongated tail that extends to the northwest, with traces of cold dust in the tail, which they suspect might be caused by a recent AGN outburst.

In order to detect these blue BCGs, we would have to relax the condition on the color. However, this condition is necessary in order to remove most of the spiral galaxies, and we find that allowing galaxies with blue colors would make the method much less reliable as the red sequence would be ill-defined. Our method is thus only reliable to detect red BCGs, even if they are not pure ellipticals (star forming or merging galaxies, for example).

\section{Luminosity profiles}

We fit 2D analytical models on sources with GALFIT (Peng et al. 2002). Once the BCG is defined, we run SExtractor one last time to return model fit parameters in the available filter closest to the $F 606 \mathrm{~W}$ rest frame at redshift $z$ (see Fig. 5), which is at a wavelength above the $4000 \AA$ break and thus in the spectral region where we get the highest flux. The chosen filters can be found in Table 1. We note that there are 37 BCGs out of the 149 for which HST data are not available in the $F 606 \mathrm{~W}$ rest frame or redder. The reddest filter is either bluer than the $4000 \AA$ break or contains it, which means that not only are we looking at the oldest, reddest star population, but also at the youngest bluest stars. These BCGs are indicated by blue squares in the plots. The redshift distribution of all our BCGs is plotted in Fig. 2; the blue histogram represents the clusters with filters which are bluer than the $4000 \AA$ break. These clusters observed in filters that are too blue are mainly between redshifts 0.7 and 1.2.

\subsection{Masking}

We first need to mask all the neighboring sources. We take the SEGMENTATION map returned by SExtractor, and unmask the BCG (which is identified by an identification number), and also mask any blank region on the image. Because of deblending issues, it is more than likely that other objects, projected on the BCG, need to be masked.

We use sharp divided images to detect any neighboring objects that pollute the signal. Sharp divided (SD) images (see e.g., Márquez et al. 1999, 2003) are obtained by dividing the images by the median filtered corresponding images. This brings out all the small neighboring sources that may have been hidden by the luminous halo of the BCG. We run SExtractor (again) on this SD image, and mask all the objects that are farther than 0.5 arcsec from the BCG coordinates (an example is given in Fig. 8), which is the minimum distance required to avoid masking the BCG center. As can be seen in Fig. 8, the sources masked based on the SD image detection seem larger on the final mask than in the SD images, as the SD image does not show the true sizes of the objects. We apply a factor of 6 to the minor and major axes of the sources detected by SExtractor on the SD image to create our final mask. This factor allows us to include all the 

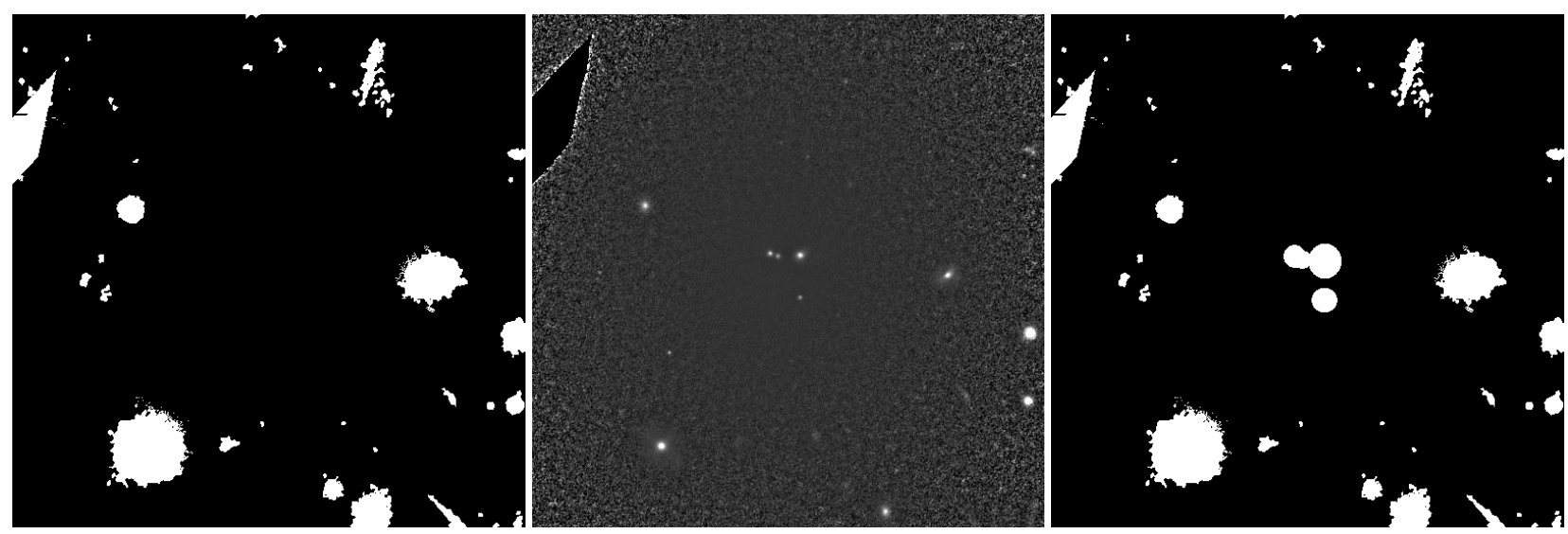

Fig. 8. Example of the BCG in the cluster Abell 2261, at $z=0.224$. Left: segmentation map returned by SExtractor, with the BCG unmasked. The pixels with a value of 1 are masked; those with a value of 0 are unmasked. Middle: sharp divided image in which four knots in the core appear. These knots were drowned in the light of the BCG and are now visible. Right: final mask (including the central objects).

luminosity of the sources and to mask them efficiently. If necessary, we identify by eye and draw the regions to be masked ourselves in SAOImage DS9 and create a new mask.

\subsection{PSF model}

To obtain a successful model of the galaxy profiles that also works for the inner regions, an accurate description of the PSF is needed. While the PSF we used for the photometry may have been sufficient to distinguish stars from galaxies, GALFIT requires the PSF to meet a number of criteria: it must have a very high $\mathrm{S} / \mathrm{N}$ and a flat and zero background (otherwise any pattern in the background will appear on the model image when convolved with the PSF); it should match the image (e.g., diffraction rings and spikes, speckle pattern); and it should be correctly centered (see GALFIT Technical FAQ).

We first subtract from the images the sky background, which is determined by masking all sources and blank areas on the cluster image, using the routine calc_background with a $3 \sigma$ clipping method. We then use PSFex, and make a selective sample of the stars that will go into making the PSF. We select all point-like sources with FLAGS $=0$, MAG_AUTO $\leq 21$, ELONGATION $\leq 1.1$, CLASS_STAR $\geq 0.98, S / N \geq 20$, and an isophotal print ISOAREA_IMAGE $\geq 20$ pixels.

Since we work on HST observations that cover a small field of view, there may not be many bright stars in the field of the cluster that we could use to compute a PSF. We tried to take several faint stars and stack them to increase the S/N of the PSF. However, we find that this often results in a PSF with an uneven background that stands out during the model fitting returned by GALFIT, and this usually ends up being a bad fit (too large effective radius, large uncertainties). Since we are working on space observations, the PSF does not vary much, and though it may vary with time, the variations should not be significant (see Martinet et al. 2017). This means that we can replace the PSF for a given filter by another one in the same filter with a better S/N. Higher S/N PSFs return better fits.

Modeled and theoretical PSFs are available for ACS/WFC and WFC3/IR. However, according to the GALFIT Technical $\mathrm{FAQ}^{5}$, the profiles obtained with models may not be realistic for space-based images, so we prefer to use observed PSFs.

\footnotetext{
https://users.obs. carnegiescience.edu/peng/work/ galfit/TFAQ.html
}

\subsection{Profile fitting}

We use GALFIT to fit two different models to our BCGs: a single Sérsic component or two Sérsic components, to allow different contributions from the inner and outer parts of the galaxies. We also tried to apply other models or combinations of models including a de Vaucouleurs profile, but they always provided worse results (i.e., they gave a worse $\chi^{2}$, and about $30 \%$ of the BCGs were not well fitted with one or two de Vaucouleurs profiles), so here we only discuss the results with Sérsic fittings.

It is necessary to give GALFIT an estimate of all the initial parameters: the effective surface brightness or total magnitude, the effective radius (the radius at which half of the total light of the galaxy is contained), and the elongation or the position angle (PA) of the BCG. These initial guesses are taken from the SExtractor catalogs: MU_EFF_MODEL or MAG_AUTO, FLUX_RADIUS, ELONGATION, and THETA_IMAGE. We did not have an estimate of the BCG Sérsic index, so we started from the value corresponding to the de Vaucouleurs profile: $n=4$. If the fitting does not converge, we try different Sérsic indices in the range 0.5-10. For the second Sérsic component that accounts for the inner part, the following parameters are considered: MU_EFF_SPHEROID, SPHEROID_REFF_IMAGE, SPHEROID_SERSICN, and SPHEROID_THETA_IMAGE. The suffix SPHEROID refers to the bulb component when SExtractor tries to model a disk and a bulb to a galaxy. We consider an elongation (minor-to-major axis ratio, $b / a$ ) of 0.90 for the inner part, as an initial guess. The region to fit is a box that is $2.5 r_{\text {Kron }}$ wide (cf. GALFIT FAQ), $r_{\text {Kron }}$ being the Kron radius returned by SExtractor. This is large enough to contain all the light from the BCG as well as some sky background, and is a good compromise to obtain good fits of our galaxies.

We first run GALFIT to fit the BCGs with one Sérsic component. If it does not manage to converge with a Sérsic index of $n=4$, we try different values between 0.5 and 10 until it converges to a meaningful fit, and reject any fit with returned effective radius larger than half the size of the fitting region, which is to say $R_{\mathrm{e}} \leq 2.5 r_{\mathrm{Kron}} / 2$ pixels. We then use the output parameters as initial guesses to fit the outer part of the galaxy and add another Sérsic component to fit the inner part of the galaxy. If it does not converge towards meaningful values, we increment the Sérsic index until it manages to fit the BCG. For pairs of BCGs (two brightest cluster galaxies with similar sizes and magnitudes), we fit both of them simultaneously. 


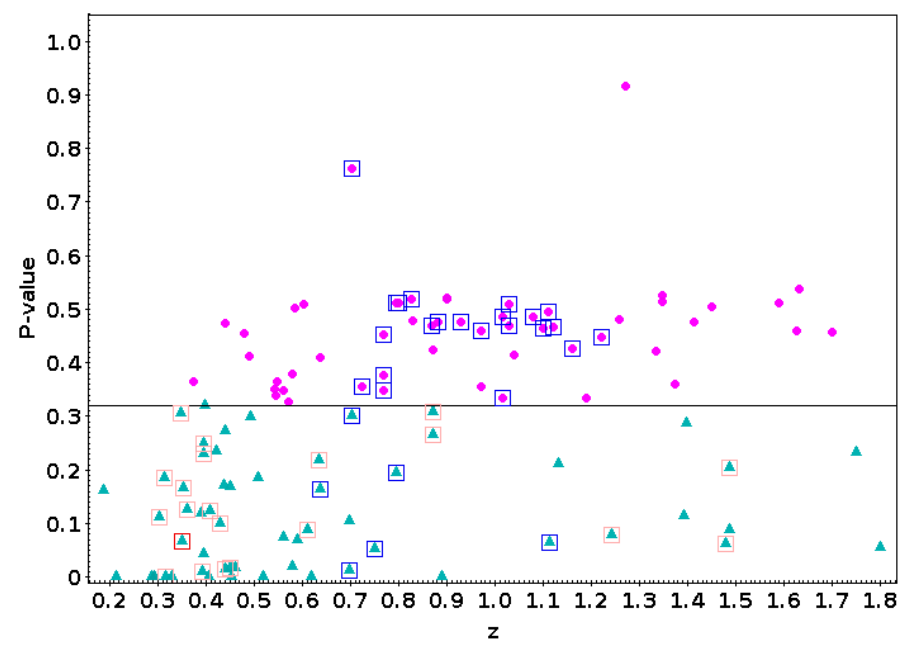

Fig. 9. Distribution of $P$-values as a function of redshift considering a model with one Sérsic component (magenta), and a model with two Sérsic components (cyan). BCGs that could not be fitted by either model are not included.

\subsection{Choice of best fit model}

The quality of the fit can be estimated from the reduced $\chi^{2}$ $\left(\chi_{v}^{2}\right)$, which should be close to 1 . From our results we find that $\chi_{v}^{2}>1.2$ and $\chi_{v}^{2}<0.8$ often indicate a bad fit. This happens when the model used to fit the BCG is not adapted, or when the initial parameters given are bad estimates. In this case, GALFIT may also not have converged and/or crashed. To decide whether a second component is really necessary to fit the BCG, or if one component gives equally good results, we use the F-test (Simard et al. 2011; Margalef-Bentabol et al. 2016). As stated in Bai et al. (2014), as the background noise is not Gaussian, the meaning of $\chi_{v}^{2}$ is not as significant, and when comparing two models a $\chi_{v}^{2}$ closer to unity does not necessarily mean that it is a better fit. So we prefer to use an F-test.

The F-test states that if the $P$-value, determined from the Fvalue and the number of degrees of freedom, is lower than a probability $P_{0}$, then we can reject the null hypothesis and consider that the second model gives a significantly better result than the simpler one. The F-value is defined as the ratio of the reduced chi-squared values of the two models. GALFIT returns the $\chi^{2}$ and the $\chi_{v}^{2}$ of the fit, but instead of directly considering the output $\chi_{v}^{2}$ computed by GALFIT, we compute $\chi_{v}^{2}$ as

$\chi_{v}^{2}=\frac{\chi^{2}}{n_{\text {d.o.f. }}}$

with $n_{\text {d.o.f. }}$ the number of degrees of freedom, which is defined here as the number of resolution elements, $n_{\text {res }}$, minus the number of free parameters in the model, $n_{\text {free }} . n_{\text {res }}$ can be calculated as (see Simard et al. 2011; Margalef-Bentabol et al. 2016)

$n_{\mathrm{res}}=\frac{n_{\text {pixels }}}{\pi \theta^{2}}$,

where $n_{\text {pixels }}$ is the number of unmasked pixels used for the fitting, and $\theta$ is the full width at half maximum (FWHM) of the given PSF in units of pixels, and $n_{\text {d.o.f. }}$ is then

$n_{\text {d.o.f. }}=n_{\text {res }}-n_{\text {free }}-1$.

The $P$-value is then calculated with the routine f.cdf from scipy.stats in python. We set $P_{0}=0.32$, which represents a $1 \sigma$ threshold value (Margalef, priv. comm.).

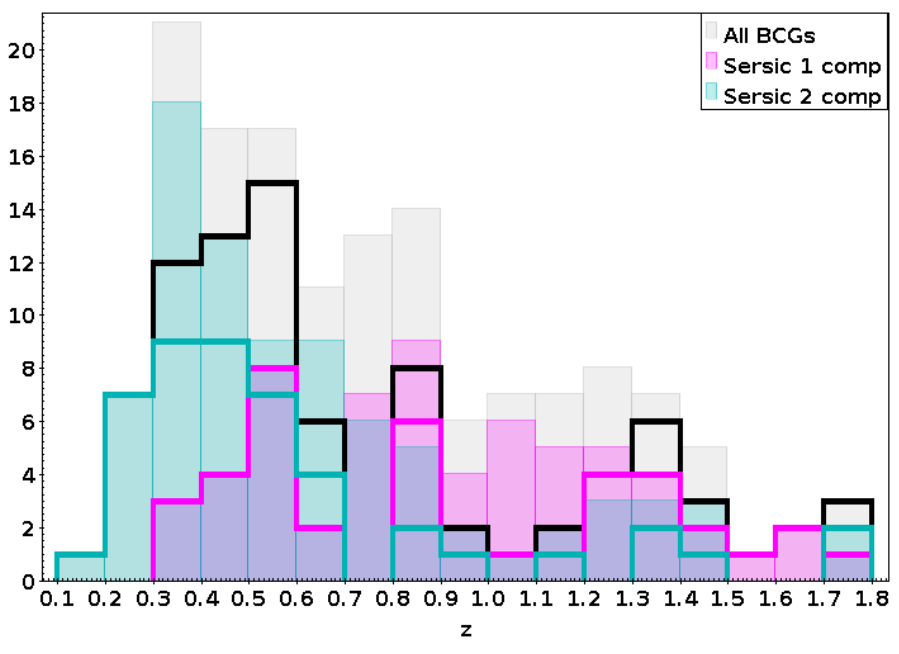

Fig. 10. Distribution of redshifts for each model: a single Sérsic component (magenta) and two Sérsic components (cyan). The overall distribution is shown in gray. The semi-filled histograms represent the initial sample, and the unfilled histograms only contain BCGs with appropriate data.

We show the distribution of $P$-values as a function of redshift in Fig. 9. A $P$-value $\leq P_{0}$ means that we need a second component to correctly model the BCG light distribution. We do not represent in this plot the BCGs that could not be fitted by either model: 9 BCGs could only be fitted with a single component, 22 could only be fitted with two components, 2 could only be fitted by fixing the Sérsic index $n=4$ (de Vaucouleurs profile), and 2 BCGs could not be fitted or returned a poor fit for either of the models. In Fig. 10 it appears that BCGs that need a second component to obtain a good fit tend to be at lower redshifts (peak at $z=0.3$ ), while the distribution for those that were well fitted with a single component is flatter. We also find BCGs with a model with two Sérsic profiles at higher redshifts (14 BCGs at $z \geq 1.0$ ). If the chosen model depended on the distance, we would have expected not to have two component BCGs at higher $z$, which is not the case.

We must remember that part of our sample ( 37 BCGs) is studied in a too blue rest frame filter, and for these clusters we are not looking at the same star population. Without taking into account those observed in too blue filters, we find that 55 out of 72 BCGs $(76 \%)$ at redshift $z \leq 0.8$ need a second component, while the trend is reversed at $z>0.8$, as 23 out of 38 BCGs $(61 \%)$ can be nicely modeled with only one component. We also find that most of the BCGs observed with too blue filters $(62 \%)$ can be modeled with only one Sérsic.

We also want to discover if the existence of these two distinct populations (BCGs with two components at low $z$, and BCGs with a single component throughout redshift), with a limit around redshift $z=0.8$, may be due to the fact that BCGs at higher redshifts are less resolved than their lower redshift counterparts. To test this hypothesis, we bring a sample of 44 BCGs at redshifts $z \leq 1.0$ to a common physical scale at redshift $z=1.2$. We smooth the images with a Gaussian and repeat the previous steps. The $\sigma_{\text {gauss }}$ of the Gaussian to apply is calculated as

$\left.\sigma_{\text {gauss }}=\sqrt{(} \sigma_{z=1.2}^{2}-\sigma_{z, \text { cluster }}^{2}\right)$

with $\sigma_{z \text {,cluster }}$ computed from the FWHM of the image we want to degrade, and $\sigma_{z=1.2}$ the $\sigma$ at the reference redshift $z=1.2$, which was computed as 
$\sigma_{z=1.2}=\sigma_{z \text {,cluster }} * \frac{\text { pixscale }_{z=1.2}}{\text { pixscale }_{z, \text { cluster }}}$.

Of the 44 BCGs at $z \leq 1.0$ on which we did this test, 30 returned results similar to those obtained with the original (unsmoothed) images. We also found that seven BCGs that were better fitted with two Sérsic components can be modeled just as well, according to the F-test, with only one Sérsic after smoothing the images. Surprisingly, the opposite also happened for seven other BCGs: four could not initially be fitted with two components and the other three were close to the $P$-value limit, $P_{\lim }=0.32$. As $68 \%$ of the tested BCGs showed no significant difference, we can confirm that the lack of resolution for the farthest BCGs does not cause the absence of an inner component for BCGs at higher redshifts.

\subsection{BCGs observed in too blue filters}

In all that follows, when considering together the results from BCGs better fit with one or two Sérsic components, we consider the values obtained for the outer Sérsic component $\left(R_{\mathrm{e}, \text { out }} \geq\right.$ $\left.R_{\mathrm{e}, \text { in }}\right)$.

As stated before, we have 37 BCGs observed in too blue filters (relative to the $4000 \AA$ break). We must determine if they can be taken into account in our final study. For this, we run a test on 40 clusters with filters available on the blue side of the $4000 \AA$ break as well as appropriate red filters, to check if the returned parameters vary depending on the filters chosen. We find that the absolute magnitude and mean effective surface brightness become fainter as the filter gets bluer. However, the dispersion is too big to simply correct for the offset to bring the BCGs observed in too blue filters to the appropriate red ones, perhaps because the filters on the blue side of the break do not always fall in the same spectral region on the SED (as the SED varies with redshift, and not all clusters were observed with the same filters). The effective radii can have their sizes halved when observed with too blue filters. As for the Sérsic indices, we find that the BCGs that need a second component tend to have Sérsic indices in bluer filters consistent with those measured in the appropriate red filters. The BCGs which could be fitted with only one Sérsic have indices that vary without any clear pattern. These observations show that we cannot directly consider together the measurements obtained looking at different parts of the SED. Therefore, we chose to exclude the BCGs observed in too blue filters in what follows.

We find however that the position angles (PA) of the BCGs are not affected and remain consistent regardless of the filter chosen (see Fig. 11). The PAs of these BCGs will thus be kept. Only one point presents a big difference between the two values $\left(\mathrm{PA}_{\text {red }}-\mathrm{PA}_{\text {blue }}>120^{\circ}\right)$. We found that the ICL associated with this $\mathrm{BCG}$ is more extended in the reddest filter; Ellien et al. (2019), also show that the ICL tends to be more extended in redder filters. The other BCGs with a significant difference between the values measured in the two different filters are circular in shape $(b / a>0.80)$, so the PAs are ill-defined, which also explains the huge error bars.

\section{Results}

As explained above, we tried fitting the BCGs with one or two Sérsic profiles. In the following the values plotted are those from the best model determined using the F-test (see previous section). The resulting parameters are summarized in Tables 2 and 3 .

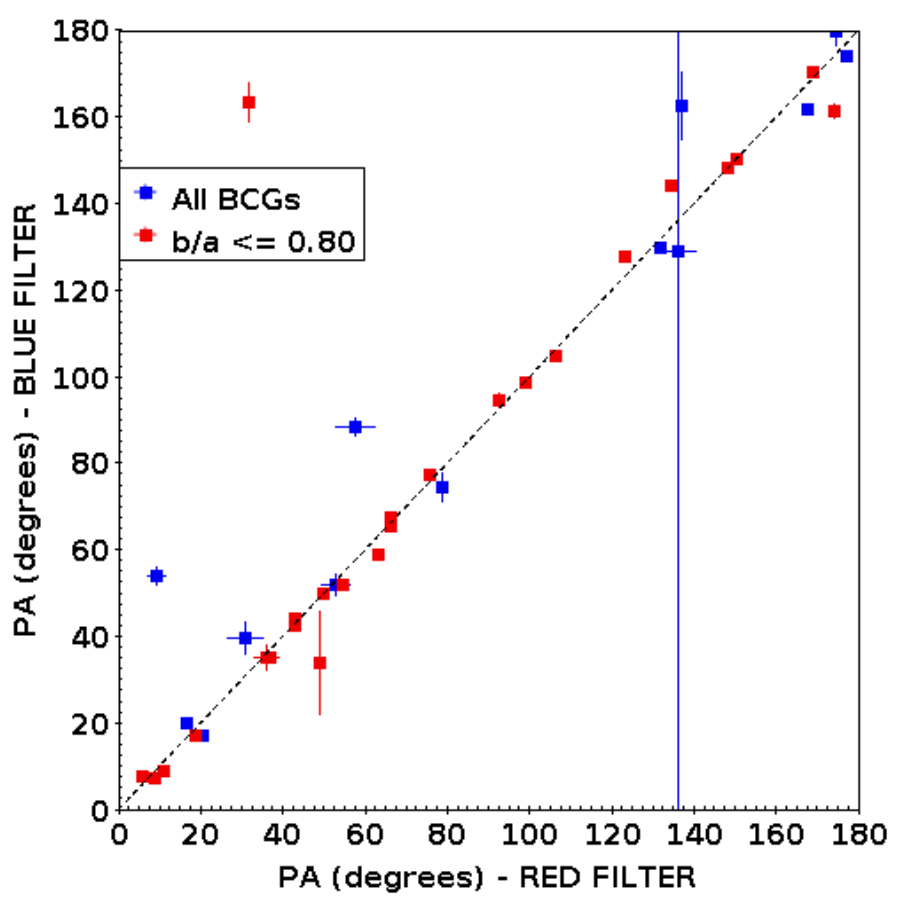

Fig. 11. PA measured in the appropriate red filter ( $x$-axis), and measured in a too blue filter ( $y$-axis). In red are BCGs with elongations $b / a \leq$ 0.80 .

Two BCGs were not properly fitted by either model, but were correctly fitted by fixing the Sérsic index $n=4$ (corresponding to a de Vaucouleurs profile). We thus kept the parameters obtained with this fit. Two other BCGs were not correctly fitted by either model, and were thus excluded, bringing our sample size to 147 BCGs.

We summarize the total number of galaxies that were fit with each model:

- Sérsic (1 component): 63 BCGs;

- Sérsic + Sérsic (2 components): 84 BCGs.

Without taking into account the BCGs observed in too blue filters we have:

- Sérsic (1 component): 40 BCGs;

- Sérsic + Sérsic (2 components): 70 BCGs.

In all the plots shown in this paper, the BCGs better fitted with two Sérsic components will be represented with triangles, and those fitted with only one component with diamonds.

Before drawing conclusions, we need to know if we can consider together the results from BCGs better fit with one and with two Sérsic components. In principle, the two subsamples can be put together if, for the two components, we assume that the outer component contains most of the light of the BCG and that the outer profile represents well enough the overall luminosity of the galaxy. The more important the contribution of the inner component to the total luminosity of the BCG is, the less accurate this statement will be. If an inner component is required to model the $\mathrm{BCG}$, then the resulting outer profile obtained when fitting two components may not be comparable to a profile obtained with only one component.

We show the histogram of the ratio of the inner component to total fluxes for the 70 BCGs requiring two Sérsic components (see Fig. 12), and find that 24 BCGs present a very important inner component, which can contribute up to $30 \%$ of the total luminosity of the galaxy. If we choose to ignore these 24 BCGs, no obvious difference can be seen in the overall 
Table 2. Parameters obtained from fitting the luminosity profiles of the BCGs with GALFIT.

\begin{tabular}{|c|c|c|c|c|c|c|c|c|c|}
\hline Name & Class & Model & $\begin{array}{l}M_{\mathrm{ABS}} \\
(\mathrm{mag})\end{array}$ & $\begin{array}{l}\left\langle\mu_{\mathrm{e}}\right\rangle \\
\left(\mathrm{mag} \operatorname{arcsec}^{-2}\right)\end{array}$ & $\begin{array}{l}R_{\mathrm{e}} \\
(\mathrm{kpc})\end{array}$ & $n$ & $b / a$ & $\begin{array}{l}\text { PA } \\
\text { (degrees) }\end{array}$ & $\begin{array}{l}\text { Alignment } \\
\text { (degrees) }\end{array}$ \\
\hline SpARCS-J0335 & 1 & Sérsic & -26.088 & 25.034 & 57.488 & 8.7 & 0.88 & 158 & 4 \\
\hline XDCPJ0044-2033 & 1 & Sérsic & -25.073 & 23.119 & 12.944 & 3.39 & 0.36 & 11 & 63 \\
\hline CLJ0152-1357 & 1 & Sérsic & -24.859 & 23.471 & 21.802 & 3.59 & 0.7 & 49 & \\
\hline CLJ015244.18-135715.84 & 2 & Sérsic & -24.378 & 22.698 & 12.74 & 4.24 & 0.75 & 42 & \\
\hline CLJ015244.18-135715.84 & 1 & Sérsic & -24.478 & 22.343 & 11.331 & 7.96 & 1.0 & 10 & \\
\hline RCSJ0220-0333 & 1 & Sérsic & -24.511 & 23.111 & 10.804 & 4.08 & 0.75 & 24 & 64 \\
\hline RCSJ0221-0321 & 1 & Sérsic & -23.916 & 22.257 & 5.667 & 0.88 & 0.61 & 111 & 63 \\
\hline RCSJ0221-0321 & 2 & Sérsic & -24.566 & 23.098 & 11.261 & 5.43 & 0.74 & 21 & 27 \\
\hline XLSSJ0223-0436 & 1 & Sérsic & -24.675 & 23.103 & 8.146 & 4.52 & 0.74 & 47 & 69 \\
\hline$\ldots$ & $\ldots$ & $\ldots$ & $\ldots$ & $\ldots$ & $\ldots$ & $\ldots$ & $\ldots$ & $\ldots$ & $\ldots$ \\
\hline SPT-CLJ0000-5748 & 1 & Sérsic2 & -25.601 & 24.306 & 47.94 & 1.85 & 0.53 & 162 & 8 \\
\hline $\mathrm{ACO} 2813$ & 1 & Sérsic2 & -24.891 & 23.516 & 49.81 & 4.7 & 0.72 & 175 & \\
\hline ACO 2813 & 2 & Sérsic2 & -24.894 & 23.362 & 46.464 & 1.3 & 0.53 & 148 & \\
\hline RXJ0056-27 & 1 & Sérsic2 & -24.641 & 23.613 & 28.883 & 1.83 & 0.64 & 94 & \\
\hline SPT-CLJ0102-4915 & 1 & Sérsic2 & -24.87 & 22.478 & 13.894 & 1.62 & 0.87 & 118 & 29 \\
\hline SPT-CLJ0102-4915 & 2 & Sérsic2 & -25.753 & 22.069 & 15.653 & 1.26 & 0.57 & 134 & 13 \\
\hline RXJ0110+19 & 1 & Sérsic2 & -24.411 & 22.709 & 26.043 & 1.1 & 0.72 & 44 & \\
\hline Abell209 & 1 & Sérsic2 & -24.327 & 21.734 & 19.715 & 2.05 & 0.71 & 134 & 23 \\
\hline SPT-CLJ0205-5829 & 1 & Sérsic2 & -25.701 & 26.197 & 82.853 & 1.09 & 0.33 & 32 & 8 \\
\hline XMMXCSJ022045.1-032555.0 & 1 & Sérsic2 & -24.403 & 21.638 & 16.486 & 2.34 & 0.91 & 65 & \\
\hline$\ldots$ & $\ldots$ & $\ldots$ & $\ldots$ & $\ldots$ & $\ldots$ & $\ldots$ & $\ldots$ & $\ldots$ & .. \\
\hline
\end{tabular}

Notes. Only the parameters obtained for the chosen model are shown. If fitted by two Sérsic profiles, the parameters of the outer component are given (the parameters for the inner component are then given in Table 3). The columns are: full cluster name, class of the galaxy, best model (Sérsic is a model with a single component, Sérsic2 is a model with two components, and Sérsic* fixes the Sérsic index $n=4$ ), absolute magnitude, mean effective surface brightness, effective radius, Sérsic index, elongation (ratio of the major to minor axis), position angle, alignment of the BCG with its host cluster. The full table is available at the CDS.

Table 3. Parameters obtained for the inner component, for BCGs fitted with two Sérsic profiles.

\begin{tabular}{llllllll}
\hline \hline Name & Class & $\begin{array}{l}M_{\text {ABS,inn }} \\
(\mathrm{mag})\end{array}$ & $\begin{array}{l}\left\langle\mu_{\mathrm{e}, \text { inn }}\right\rangle \\
\left(\mathrm{mag} \mathrm{arcsec}^{-2}\right)\end{array}$ & $\begin{array}{l}R_{\mathrm{e}, \text { inn }} \\
(\mathrm{kpc})\end{array}$ & $n_{\text {inn }}$ & $b / a_{\text {inn }}$ & $\begin{array}{l}\mathrm{PA}_{\text {inn }} \\
(\mathrm{degrees})\end{array}$ \\
\hline SPT-CLJ0000-5748 & 1 & -23.363 & 22.079 & 6.133 & 1.18 & 0.66 & -6 \\
ACO2813 & 1 & -22.077 & 19.314 & 1.968 & 0.37 & 0.76 & -3 \\
ACO2813 & 2 & -23.127 & 21.373 & 8.236 & 2.03 & 0.87 & -45 \\
RXJ0056-27 & 1 & -23.153 & 22.119 & 7.314 & 4.47 & 0.89 & -32 \\
SPT-CLJ0102-4915 & 1 & -23.625 & 19.466 & 1.956 & 1.6 & 0.73 & -12 \\
SPT-CLJ0102-4915 & 2 & -24.621 & 19.962 & 3.521 & 1.54 & 0.47 & -48 \\
RXJ0110+19 & 1 & -23.367 & 19.475 & 3.634 & 2.32 & 0.92 & 23 \\
Abell209 & 1 & -20.251 & 17.611 & 0.452 & 1.28 & 0.93 & 68 \\
SPT-CLJ0205-5829 & 1 & -24.978 & 23.76 & 19.333 & 7.67 & 0.72 & -19 \\
XMMXCSJ022045.1-032555.0 & 1 & -21.69 & 18.129 & 0.939 & 1.51 & 0.72 & 67 \\
.. & $\ldots$ & $\ldots$ & $\ldots$ & $\ldots$ & $\ldots$ & $\ldots$ & $\ldots$ \\
\hline
\end{tabular}

Notes. The columns are: full cluster name, class of the galaxy, absolute magnitude, mean effective surface brightness, effective radius, Sérsic index, elongation (ratio of the major to minor axis), position angle. The full table is available at the CDS.

relations observed in the following. However, we prefer to exclude them as the outer profile may not be comparable to the profile obtained with a single component modeling most of the light of the galaxy. After excluding the galaxies with a very bright inner component and those observed in too blue filters, we obtain the final numbers:

- Sérsic (1 component): 40 BCGs;

- Sérsic + Sérsic (2 components): 46 BCGs.

In the following plots, BCGs observed in too blue filters are indicated by blue squares, and those fit with two Sérsic profiles and with an important inner component are indicated by light pink squares. We also identify the blue SF BCGs (cf. Sect. 3) with red squares and pairs of BCGs with black triangles.

\subsection{Evolution with redshift}

In order to study the evolution of the BCGs, we consider the dependence of the derived parameters as a function of redshift.

The absolute magnitudes of the BCGs, computed from the total apparent magnitudes (see Table 1 for the filters considered) 


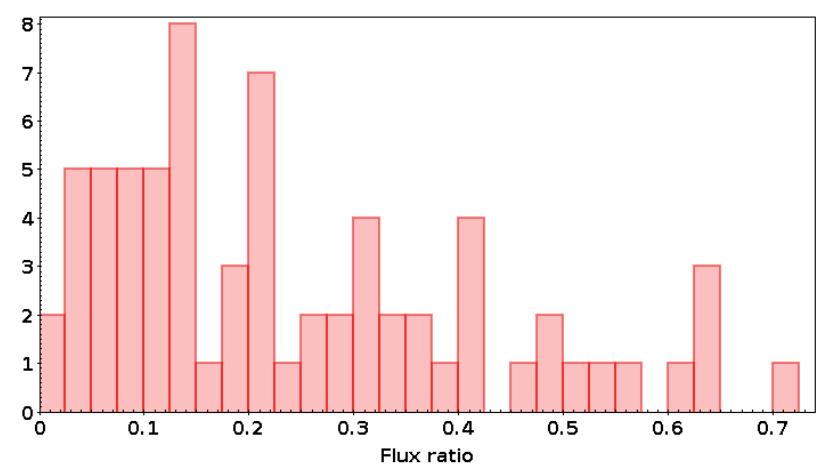

Fig. 12. Histogram of the ratio of the flux of the inner component to the total flux of the BCG, for clusters better fit with two components. Clusters observed in too blue filters are excluded in this plot.

calculated by GALFIT, despite the very big dispersion (4 mag thoughout redshift) tend to become brighter with redshift (see Fig. 13, left). The trend is faint, and can be quantified with a correlation coefficient $R=-0.29$ and a $p$-value $p=0.006563$ (calculated from the coefficient $R$ and the number of data points $N^{6}$ ). By taking a significance level of $\alpha=0.05$, we show that we can reject the null hypothesis $(p<\alpha)$ and conclude that the trend is significant.

There is a moderate trend for BCGs to grow with time (Fig. 13, right), as those with the smallest effective radii are at higher redshifts $(z \geq 1.2)$. The trend in logarithmic scale is quantified by a correlation coefficient $R=-0.40$, and with a $p$-value of $p=0.000142$. BCGs observed in too blue filters generally have smaller effective radii than the others at a given redshift Those with an important inner component contribution do not appear to occupy a special place in these relations.

The mean effective surface brightness (Fig. 14) shows no significant evolution as a function of redshift $(R \ll 0.1)$, with a very large dispersion (spanning $6 \mathrm{mag}$ at $z \geq 1.25$ ). Seven BCGs at higher redshifts $(z \geq 1.4)$ can be seen among the galaxies with the brightest mean effective surface brightnesses $\left(\langle\mu\rangle \leq 22 \mathrm{mag} \operatorname{arcsec}^{-2}\right)$. We confirm that nothing peculiar was observed with these BCGs. Those observed in too blue filters and those with an important inner component contribution do not occupy a specific place in the relation.

The vertical gradient in color in Fig. 14 shows that the large dispersion is also linked to the effective radius. As we go towards the biggest BCGs (increasing effective radii), the relation is shifted towards the fainter mean effective surface brightnesses. This is to be linked with the Kormendy relation, which is shown in Sect. 5.2.

Finally, there is no correlation between the Sérsic index and redshift (Fig. 15, left). However, in the right panel, we see two different populations: the BCGs that were modeled with only one component generally have high Sérsic indices with a strong peak at $n_{1 \text { comp,mean }}=4$ (without considering the BCGs observed in too blue filters), while the BCGs that were better modeled with two Sérsic components with lower Sérsic indices show a peak at $n_{2 \text { comp,mean }}=2.0$.

\subsection{Kormendy relation}

The Kormendy relation (Kormendy 1977) links the (mean) effective surface brightness of elliptical galaxies to their effective

\footnotetext{
6 https://www. socscistatistics.com/pvalues/ pearsondistribution. aspx
}

radius. This relation is plotted in Fig. 16. The different colors represent different redshift bins: $z \leq 0.3,0.3<z \leq 0.5$, $0.5<z \leq 0.9,0.9<z \leq 1.3$, and $z>1.3$. We show that all the BCGs seem to follow the Kormendy relation with the same slope, but the ordinate at the origin of the line decreases with increasing redshift.

While applying a linear regression to the relation obtained in each redshift bin $(R>0.80, p<\alpha)$, we find that the slope remains quite constant at all redshift bins, $m=3.33 \pm 0.73$, whereas the ordinate at the origin varies as $c=2.15 * z+16.65$.

\subsection{Inner component}

The sample requiring an inner component consists of 46 BCGs. We find that the inner component follows a Kormendy relation (Fig. 17), and is a continuation of the Kormendy relation shown in Fig. 16 at brighter mean effective surface brightness and smaller effective radius $\left(R_{\mathrm{e}, \text { inn }} \leq 20 \mathrm{kpc}\right)$.

We observe a very faint trend for the inner components to have brighter surface brightnesses with decreasing redshift, but the trend is not significant $(R=0.27, p=0.06237>\alpha)$. We do not find any clear correlation (correlation coefficient $\leq 0.2$ ) between redshift and the absolute magnitude, effective radius, or Sérsic indices of the inner component of the BCGs.

\subsection{Alignment of the BCG with its host cluster}

Some studies have shown that BCGs tend to have a similar orientation, or PA, to that of their host cluster (West et al. 2017; Durret et al. 2019). As a comparison, we reproduce this study and compare our results with those of these two papers. The PA of the host clusters are taken from West et al. (2017) (computed from the moments of inertia of the distribution of red sequence galaxies) and Durret et al. (2019) (computed from density maps of red sequence galaxies), and the PAs of the BCGs are measured here with GALFIT. If measurements are given in both papers, the $\mathrm{PA}_{\text {cluster }}$ in Durret et al. (2019) was used, unless the $\mathrm{PA}_{\text {cluster }}$ is illdefined (when the clusters are circular in shape), in which case the PA from West et al. (2017) was used. We did not measure the PA of the host clusters for the clusters that are not presented in the above-quoted papers as our images are not large enough to accurately measure the full extent and shape of the cluster.

We include all BCGs for which the PA of the cluster was measured (73 BCGs), including those observed with too blue filters, as the PA measured by GALFIT is the same regardless of the filter (see Fig. 11). We show the histogram of the alignment between the BCGs and their host clusters (defined as the difference of PA between that of the cluster and the BCG) in Fig. 18.

We find that 39 BCGs (53\%) are aligned with their host cluster with a difference smaller than $30^{\circ}$. This already shows a tendency for BCGs to align with their host clusters, as a random orientation of the BCGs would result in a flat distribution. BCGs with the highest PA difference tend to be circular in shape (elongation $=b / a \approx 1$, for which it is more difficult to measure a $\mathrm{PA}$, resulting in high uncertainties). We thus chose to exclude all BCGs with axis ratio $b / a \geq 0.8$, in order to eliminate BCGs with ill-defined PAs, as shown in red in the histogram. We then find that 32 out of 58 of BCGs are aligned with their host cluster within $30^{\circ}$, slightly increasing the percentage to $55 \%$. There is a secondary peak between a PA difference of 30 to $40^{\circ}$, mainly corresponding to BCGs at redshift $z \geq 0.9$. At such high redshifts, galaxies appear smaller, and therefore the accuracy of the measured PA is probably worse. If we only consider galaxies at $z \leq 0.9$ (blue histogram), we find that 22 BCGs out of $30(73 \%)$ 

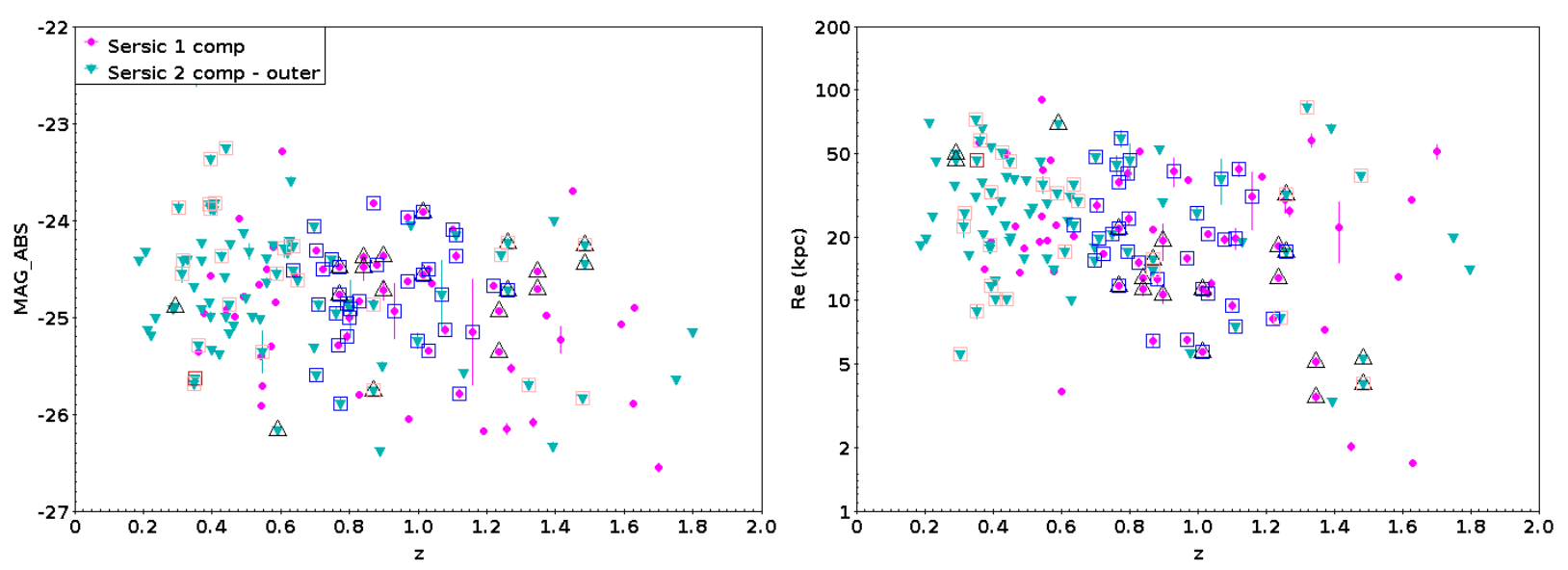

Fig. 13. Left: absolute magnitude and Right: effective radius in logarithmic scale as a function of redshift. Cyan triangles are BCGs fit by two Sérsic components (the outer component is considered), while the magenta points are BCGs fit with only one Sérsic component. Blue squares represent the BCGs observed in too blue filters, red squares are SF BCGs (see Sect. 3), black triangles are pairs of BCGs, and the light pink squares are BCGs with an important inner component contribution.

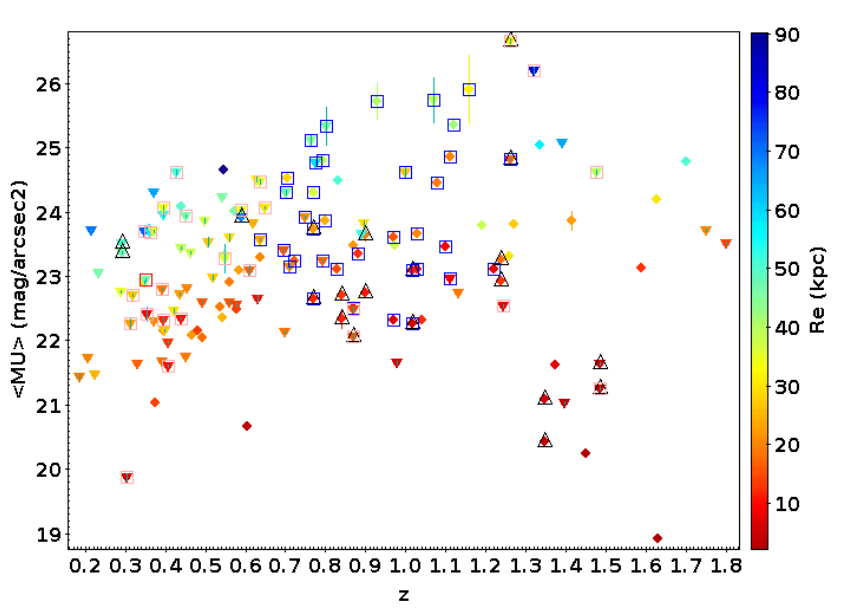

Fig. 14. Mean effective surface brightness as a function of redshift (color-coding as in Fig. 13). Additional information on the effective radii of the BCGs is shown on the right of the figure.

align with their cluster within less than $30^{\circ}$. This shows that most BCGs tend to align with their host cluster at least at $z \leq 0.9$.

\subsection{BCG physical properties as a function of host cluster properties}

We browsed the available bibliography to retrieve the cluster masses and X-ray center coordinates. The corresponding data can be found in Table 4. We preferred lensing-based mass estimates if they were available. We brought all the masses to $M_{200}$, applying the conversion factor between $M_{500}$ and $M_{200}$ : $M_{500}=0.72 M_{200}$ (Pierpaoli et al. 2003).

We show the richness of the cluster as a function of redshift and cluster mass in Fig. 19. The richness $N$ of the cluster is defined here as the number of red sequence galaxies (found in Sect. 3) in an aperture of $500 \mathrm{kpc}$ radius around the BCG. We obtain different values of $N$ for two different BCGs in the same cluster because the richness is computed in an aperture centered on each BCG.

As can be seen in Fig. 19 (left panel), clusters seem to become richer with decreasing redshift (correlation coefficient in logarithmic scale $R=-0.70$ and $p$-value of $p<10^{-5}$ ). Clusters at higher redshifts $(z \geq 1.0)$ have a lower richness, with a number of detected red sequence galaxies $N \leq 60$. The right panel also shows that the most massive clusters are also the richest, and the high-redshift clusters (blue points on the plot) with a low richness are also the least massive $\left(M_{200, \mathrm{c}} \leq 5 \times 10^{14} M_{\odot}\right)$. This low value of $\mathrm{N}$ could in principle be due to the depth of our images as we have a bias due to the distance: at higher redshifts it is more difficult to detect objects, and only the brightest ones can show up.

However, when looking at Fig. 20, the left panel shows that we do not observe very massive clusters at high redshifts. So we have no bias due to the distance of the galaxies when measuring cluster masses: the masses are measured via lensing or derived from X-ray or SZ maps, which are independent of distance. Thus, we conclude that clusters become richer with time, and this result is not due to the depth of our images. However, although we only observe very massive clusters at lower redshifts $\left(M_{200, \mathrm{c}} \geq 30 \times 10^{14} M_{\odot}\right.$ at $\left.z \leq 0.8\right)$, the masses of the clusters do not vary much with time $(R<0.20)$. The right panel shows that the very massive clusters only host bigger BCGs: clusters with masses $M_{200, \mathrm{c}} \geq 25 \times 10^{14} M_{\odot}$ only have BCGs with effective radii $R_{\mathrm{e}} \geq 30 \mathrm{kpc}$. We find no correlation $(R \leq 0.2)$ between the BCG surface brightnesses or Sérsic indices and the cluster masses.

Using the relation given in Bai et al. (2014), we compute an estimate of the BCG masses from the cluster masses: $M_{\mathrm{BCG}}^{*} \propto$ $M_{\text {cluster }}^{0.6}$. We find that the most massive BCGs are also the biggest (moderate correlation with $R=0.46$ and $p=0.00007$ ) and also tend to be brighter $(R=-0.32, p=007353)$. No correlation between the BCGs masses and redshift is seen $(R<0.20)$.

We also study how the BCGs behave depending on their offsets to the cluster X-ray center. We exclude superclusters and clusters that present several substructures and/or several BCGs. We show the histogram of the offsets in Fig. 21, top left panel. We find that 31 out of $61(51 \%)$ are within a $30 \mathrm{kpc}$ radius range from the X-ray center of the cluster, showing that BCGs tend to lie close to the cluster X-ray centers. The two star forming BCGs that have undergone recent mergers and are not at equilibrium are also located at the center of the cluster $\left(D_{\mathrm{X}} \leq 10 \mathrm{kpc}\right)$. We confirm, however, that there can be a significant offset between the two: 12 out of 61 BCGs (20\%) present an offset bigger than $100 \mathrm{kpc}$. Although the corresponding plots are not shown here, 

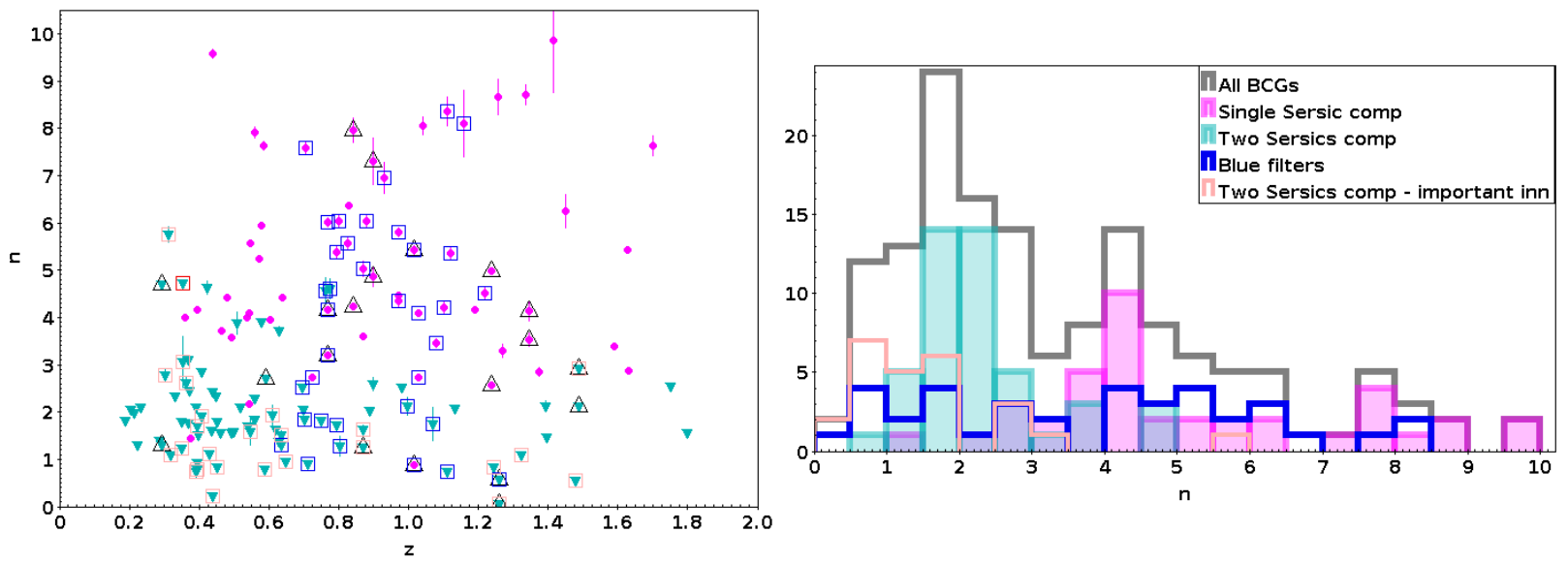

Fig. 15. Left: Sérsic indices as a function of redshift (see Fig. 13 for color-coding). Right: distribution of the Sérsic indices. All BCGs are represented in the gray histogram. The magenta and cyan histograms represent the distributions obtained with one and two components, respectively, only for the BCGs with appropriate data. The blue and lighter pink histograms represent BCGs observed in too blue filters and with an important inner component, respectively.

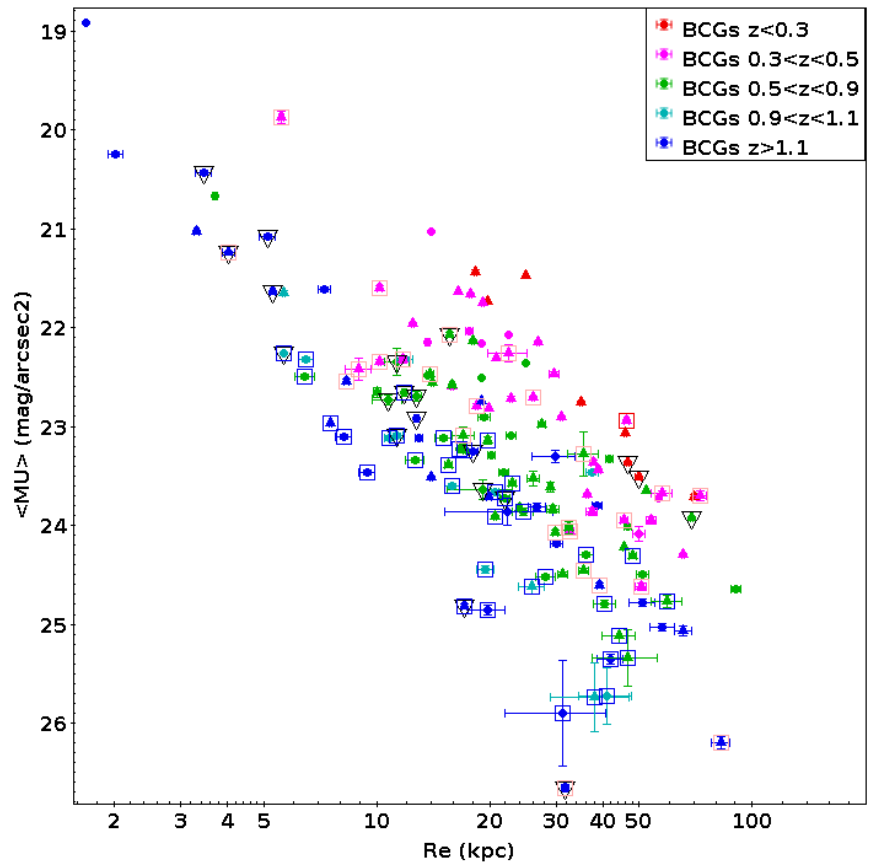

Fig. 16. Kormendy (1977) relation using the parameters obtained with one Sérsic component and the outer component of the two Sérsic component model. Different colors represent different redshift bins. Symbols are the same as in Fig. 13.

we find no correlation between the offset and the absolute magnitude, effective radius, or Sérsic index of the BCGs, or with the alignment previously computed.

As can be seen in the top right and bottom left panels of Fig. 21, however, the more massive and richer the cluster (or the BCG, as we converted the cluster masses to BCG masses), the closer the BCG is to the X-ray center of the cluster; the objects with the biggest offets $(\geq 100 \mathrm{kpc})$ have mass $M_{\text {cluster }} \leq$ $10 \times 10^{14} M_{\odot}$ and richness $N \leq 100$. We also find that there is a moderate correlation between the offset and the mean effective surface brightness of the BCG (see Fig. 21, bottom right); BCGs tend to have brighter mean effective surface brightnesses the closer they are to the X-ray center (in logarithmic scale, $R=0.34, p=0.0395)$.

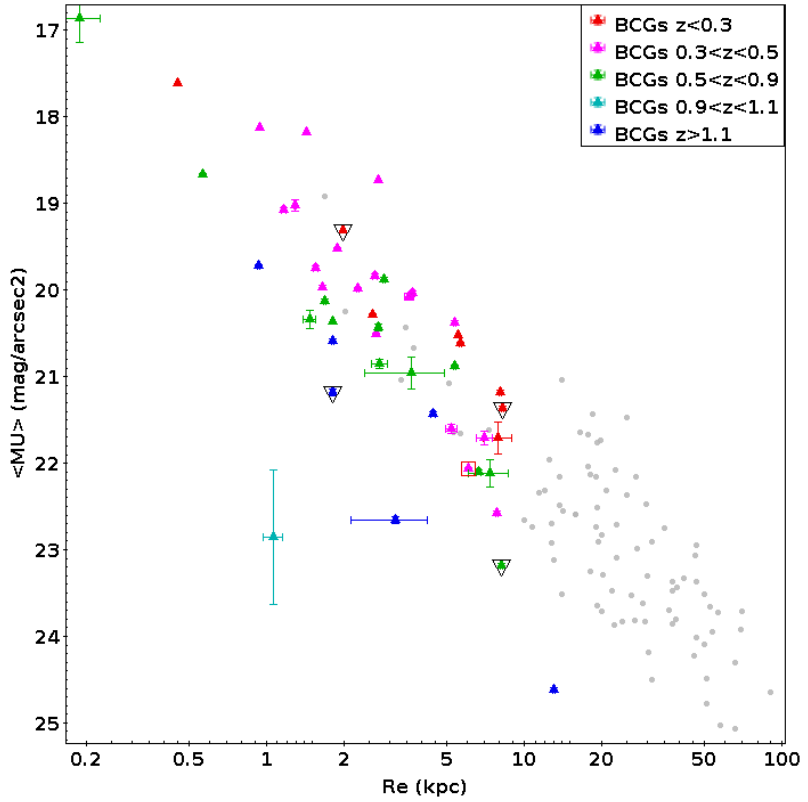

Fig. 17. Same as Fig. 16, but for the inner component of BCGs fitted with two components. The gray points are the same as in Fig. 16.

We also analyzed whether the most luminous BCGs are special (see Lin et al. 2010; Lauer et al. 2014). We studied the distribution of the difference in magnitude between the BCGs and the second ranked galaxies of the clusters. We found that the distribution was continuous, with most BCGs having a difference smaller than one magnitude with the second ranked galaxy. By selecting BCGs that are at least 1 mag brighter than the second ranked galaxy of the cluster (9 BCGs), we find that the most luminous BCGs do not occupy a specific place in the observed relations.

\section{Discussion and conclusions}

Our work deals with the largest sample (to our knowledge) of BCGs with HST imaging, covering a broad redshift interval from $z=0.1$ to $z=1.8$ (see Fig. 1), thus enabling us to trace the evolution of BCGs through time. Our sample is larger than most 


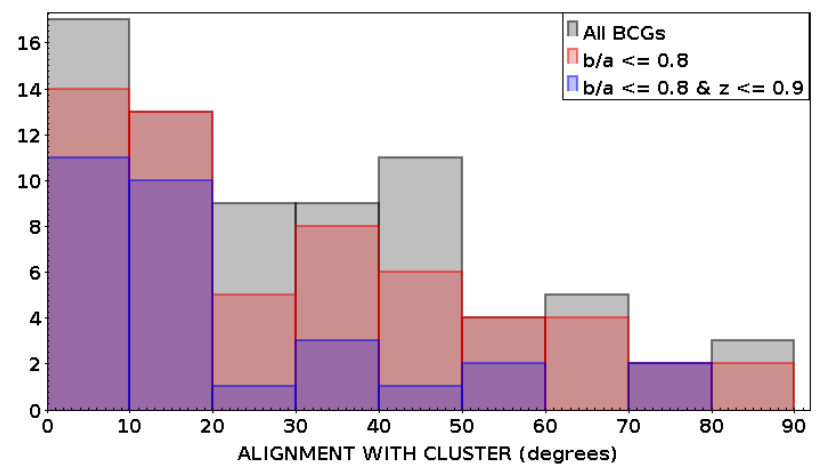

Fig. 18. Difference between the PA of the cluster (see West et al. 2017; Durret et al. 2019) and that of the BCG, as returned by GALFIT. Only clusters found in West et al. (2017) and Durret et al. (2019) are included here. The histogram is to be compared to that shown in West et al. (2017) as a way to check that our results agree with theirs. All BCGs are represented in the gray diagram, while only those with an axis ratio $b / a \leq 0.8$ are included in the red diagram. We also exclude all BCGs at redshift $z>0.9$ on the blue histogram.

studies found in the literature based on HST images, such as Bai et al. (2014), DeMaio et al. (2019), and Durret et al. (2019). We studied the luminosity profiles of these galaxies and how they evolve as a function of redshift. HST images allowed us to perform profile fitting with precision, and GALFIT returns accurate parameters from model fitting.

We developed a new tool to detect automatically red BCGs on optical images. We successfully detected all the red BCGs regardless of their peculiar characteristics (see Sect. 3). We did not manage to detect in this way the blue BCGs, which represent here only $2 \%$ of our sample.

We then proceeded to model the luminosity profiles of these automatically detected BCGs, as well as those that have only one filter available, bringing this sample to 149 BCGs. We removed all BCGs observed in too blue filters as well as BCGs better modeled with two components for which the inner component has an important contribution to the total luminosity of the galaxy. Our final sample consisted of 86 BCGs.

We studied how the photometric properties of BCGs correlate with redshift, and despite the weak but significant correlation we find that the absolute magnitude presents a faint trend of becoming brighter with time.

We show that there is a faint trend (see Fig. 13, right) for BCGs to become bigger with decreasing redshift. This is the behavior we expect for galaxies that grow in size with time by accreting gas and merging with other smaller galaxies. This trend was also observed in Durret et al. (2019) up to redshift 0.9, and can be confirmed up to redshift $z=1.8$. Based on this relation, we find that BCGs grow by more than a factor of 3 between redshifts 1.8 and 0.1 . The dispersion can be linked with the Kormendy relation: galaxies with higher surface brightnesses have smaller effective radii.

We find no strong correlation between the other photometric properties (surface brightness or Sérsic index) of the BCGs and redshift. This is in agreement with Bai et al. (2014) who do not find any correlation between the magnitude or the mean surface brightness of the BCGs and redshift, up to redshift $z=0.9$. We add that no evolution can be observed up to redshift $z=1.8$.

Although we only observe massive clusters at lower redshifts $\left(M_{200, \mathrm{c}} \geq 30 \times 10^{14} M_{\odot}\right.$ at $\left.z \leq 0.8\right)$, overall the masses of the clusters do not correlate with redshift. The growth of the cluster is mainly to be linked with the cluster richness (Fig. 19); clus- ters become richer with time, and we find that the number of red sequence galaxies in an aperture of $500 \mathrm{kpc}$ centered on the BCG increases by almost a factor of 10 between $z=1.8$ and $z=0.1$. We confirmed that the low richness we measured at higher redshift is not due to the depth of our images. This growth mainly seems to be happening at $z \leq 1.0$ as we do not observe a significant variation of the richness of the clusters before that time.

We used the relation found in Bai et al. (2014) to compute the BCG masses from the cluster masses, based on the relation found by Bai et al. (2014). We find that bigger BCGs are also more massive (see Fig. 19): $R_{\mathrm{e}} \propto 4.42 \times M_{\mathrm{BCG}}$, but the masses do not show a significant growth with redshift.

We thus find that the sizes of the BCGs grow faster than their masses in the same redshift range. Although we do not find that the masses of the BCGs grow significantly with time, whereas Bai et al. (2014) finds a factor of 2 since $z=2$, we agree that the sizes have grown significantly faster than the masses in the same redshift range. Bai et al. (2014) find that the sizes grow more than twice as fast as the masses. We confirm that the masses and sizes of BCGs do not grow at the same rate. This is in favor of a scenario in which BCGs grow thanks to minor dry mergers at the later stages of their formation and evolution. A growth mainly due to major dry mergers would indeed make the sizes and masses grow at the same rate.

To summarize, we can say that the sizes of the BCGs, as well as the richnesses of the clusters, evolve with redshift: clusters become richer with time and, at the same time, BCGs undergo dry mergers that increase their sizes.

Another interesting result is the distribution of Sérsic indices (see Fig. 15) that shows two different populations, one with low Sérsic indices mainly at low redshift $(z \leq 0.8)$ and one with high Sérsic indices. The limit is also to be linked to the fact that BCGs at lower redshifts often require a second component to correctly take into account the brighter core of the galaxy. We find that BCGs better modeled with two components have a peak Sérsic index $n=2$, while those that were fit with a single component have a peak at $n=4$. Those modeled with only one Sérsic component are thus comparable to pure elliptical galaxies which can be well modeled with a deVaucouleurs profile. This slightly differs from the results shown in Bai et al. (2014) who find a median value of $n=5.7$. However, Bai et al. (2014) only fit a single Sérsic profile to all the BCGs in their sample. If we only look at the distribution we obtained for BCGs modeled with a single component, we find that this distribution is more comparable to that of ETGs shown in their paper. Another difference with that study is related to the filters chosen to model the luminosity profiles of the BCGs. While we consider the same spectral region of the SED for all clusters in order to only look at the same old red stellar population at all redshifts, Bai et al. (2014) observe all BCGs with the ACS $F 814 W$ filter, which we find is already too blue for clusters at redshifts $z \geq 0.57$. We also showed, by studying the parameters obtained in two different filters for a sample of BCGs, that the parameters vary depending on the part of the SED you look at: when looking at a bluer filter the absolute magnitude and mean effective surface brightness become fainter, the effective radius becomes smaller, and the Sérsic indices vary without any clear trend.

Finally, we find that the Kormendy relation (Kormendy 1977) is also a function of redshift, with the relation shifted towards fainter mean effective surface brightnesses at higher redshifts. This relation shows that, at the effective radius, smaller galaxies are brighter and denser than the bigger ones. The slope of $3.33 \pm 0.73$ measured with our sample remains constant with redshift. Our value is in good agreement with that given in 
Table 4. Cluster properties.

\begin{tabular}{|c|c|c|c|c|c|c|c|c|}
\hline Name & $\begin{array}{l}\mathrm{RA}_{\text {cluster,X }} \\
\text { (J2000) }\end{array}$ & $\begin{array}{l}\text { Dec }_{\text {cluster,X }} \\
(\mathrm{J} 2000)\end{array}$ & Ref & $\begin{array}{l}M_{200, \mathrm{c}} \\
10^{14} M_{\odot}\end{array}$ & Method & Ref & $\begin{array}{l}\mathrm{PA}_{\text {cluster }} \\
\text { (degrees) }\end{array}$ & Ref \\
\hline SPT-CLJ0000-5748 & 0.25 & -57.8093 & 1 & $6.04_{-1.61}^{+1.61}$ & SZ & 1 & $170 \pm 22$ & 2 \\
\hline $\mathrm{Cl0016}+1609$ & & & & $25.76_{-6.66}^{+6.66}$ & WL & 3 & $35 \pm 0$ & 4 \\
\hline SpARCS-J0035 & 8.9588 & -43.2029 & 5 & $2.5_{-1.0}^{+0.9}$ & $\sigma-M_{200}$ & 6 & $154 \pm 8$ & 2 \\
\hline ACO2813 & 10.8519 & -20.6229 & 7 & $8.17_{-1.61}^{+1.91}$ & WL & 8 & & \\
\hline XDCPJ0044-2033 & 11.022 & -20.5665 & 9 & $3.98_{-1.58}^{+1.58}$ & $\mathrm{X}$ & 10 & $128 \pm 49$ & 2 \\
\hline RXJ0056-27 & 14.2338 & -27.67 & 11 & $2.84_{-1.59}^{+1.59}$ & WL & 3 & & \\
\hline SPT-CLJ0102-4915 & 15.734 & -49.2656 & 1 & $25.4_{-4.9}^{+4.9}$ & WL & 3 & $147 \pm 2$ & 2 \\
\hline RXJ0110+19 & 17.575 & 19.6397 & 11 & $2.36_{-1.22}^{+1.22}$ & WL & 3 & & \\
\hline Abell209 & 22.969 & -13.6108 & 12 & $9.5_{-0.7}^{+0.7}$ & Lens & 13 & $131 \pm 0$ & 4 \\
\hline CLJ0152-1357 & 28.1712 & -13.9686 & 14 & $14.0_{-4.6}^{+4.6}$ & WL & 15 & & \\
\hline$\ldots$ & $\cdots$ & $\cdots$ & $\cdots$ & $\cdots$ & $\cdots$ & $\cdots$ & $\ldots$ & $\ldots$ \\
\hline
\end{tabular}

Notes. The columns are: full cluster name, X-ray coordinates of the cluster, mass of the cluster, method used to measure the mass of the cluster, PA of the cluster. The full table is available at the CDS.

References. (1) Chiu et al. (2015); (2) West et al. (2017); (3) Sereno (2015); (4) Durret et al. (2019); (5) Fassbender et al. (2011); (6) van der Burg et al. (2014); (7) Bartalucci et al. (2019); (8) Okabe \& Smith (2016); (9) Tozzi et al. (2015); (10) Cooke et al. (2019b); (11) Hoekstra et al. (2010); (12) Postman et al. (2012b); (13) Merten et al. (2015); (14) Sayers et al. (2019); (15) Martinet et al. (2016); (16) DeMaio et al. (2019); (17) Jee et al. (2011); (18) Andreon et al. (2014); (19) Zitrin et al. (2012); (20) Stott et al. (2010); (21) Czakon et al. (2015); (22) Herbonnet et al. (2020); (23) Sereno \& Zitrin (2011); (24) Brodwin et al. (2015); (25) Chan et al. (2019); (26) Jee et al. (2005); (27) Hicks et al. (2013); (28) Just et al. (2019); (29) Hicks et al. (2008); (30) Noble et al. (2011); (31) Gonzalez et al. (2015); (32) Dahle (2006); (33) Guennou et al. (2014); (34) Jørgensen et al. (2018); (35) Santos et al. (2012); (36) Jee et al. (2017); (37) Mo et al. (2016); (38) Martini et al. (2013); (39) Richard-Laferrière et al. (2020); (40) Sereno \& Covone (2013); (41) Lidman et al. (2012); (42) Sanders et al. (2017); (43) Donnarumma et al. (2009).
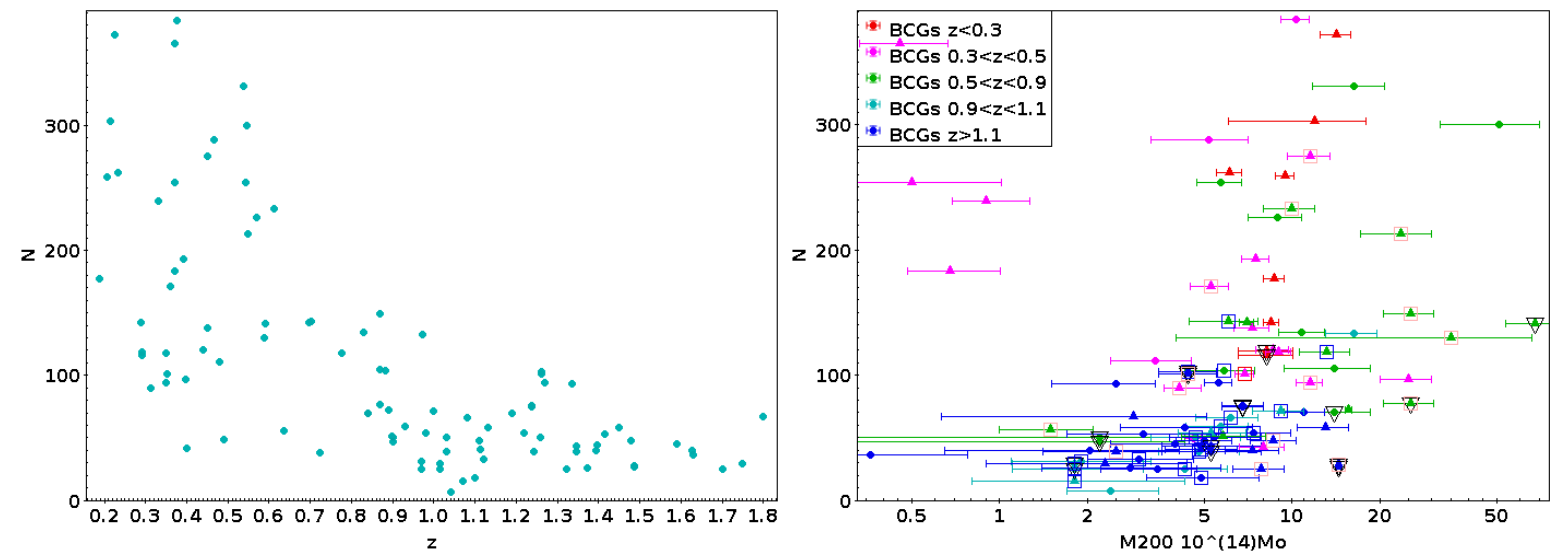

Fig. 19. Left: richness of the cluster (see text) as a function of redshift. Right: richness of the cluster as a function of its mass $M_{200, c}$. The colors represent different redshift bins.

Bai et al. (2014),

$\langle\mu\rangle=(3.50 \pm 0.18) \log R_{\mathrm{e}}+(18.01 \pm 0.23)$,

and agrees within one $\sigma$ with the value given in Durret et al. (2019),

$\langle\mu\rangle=(2.64 \pm 0.35) \log R_{\mathrm{e}}+(19.7 \pm 0.5)$.

It should be noted that cosmology or selection effects might be contributing to the results in Fig. 13, which shows a trend for BCG sizes and luminosities to increase with time. Despite its faintness, the contribution of the ICL should be taken into account. The ICL blends with the envelope of the BCG, making it difficult to differentiate the galaxy from the ICL, and this may affect our measurements (in particular those of the effective radii and Sérsic indices). The ICL might contribute at some level to measured sizes and luminosities of galaxies at low redshifts, yet might be missed in high-redshift clusters because of cosmological surface brightness dimming, or perhaps because the ICL has not yet developed in these young clusters. A concern comes from the value of the background, as GALFIT is sensitive to it, but its computation is limited by the sizes of the images (even without cropping).

We broaden the work by West et al. (2017) and Durret et al. (2019) on the alignment of BCGs with their host clusters. We removed BCGs with ill-measured position angles due to their circular shape, as well as BCGs at higher redshifts, $z \geq 0.9$, as they would appear smaller on the CCD, and would thus be less resolved and have less accurate measured PAs. This enables us to conclude that BCGs tend to align with their host cluster at least at $z \leq 0.9$, as after this selection $73 \%$ of the remaining BCGs are aligned with their host cluster within $30^{\circ}$. This is a 

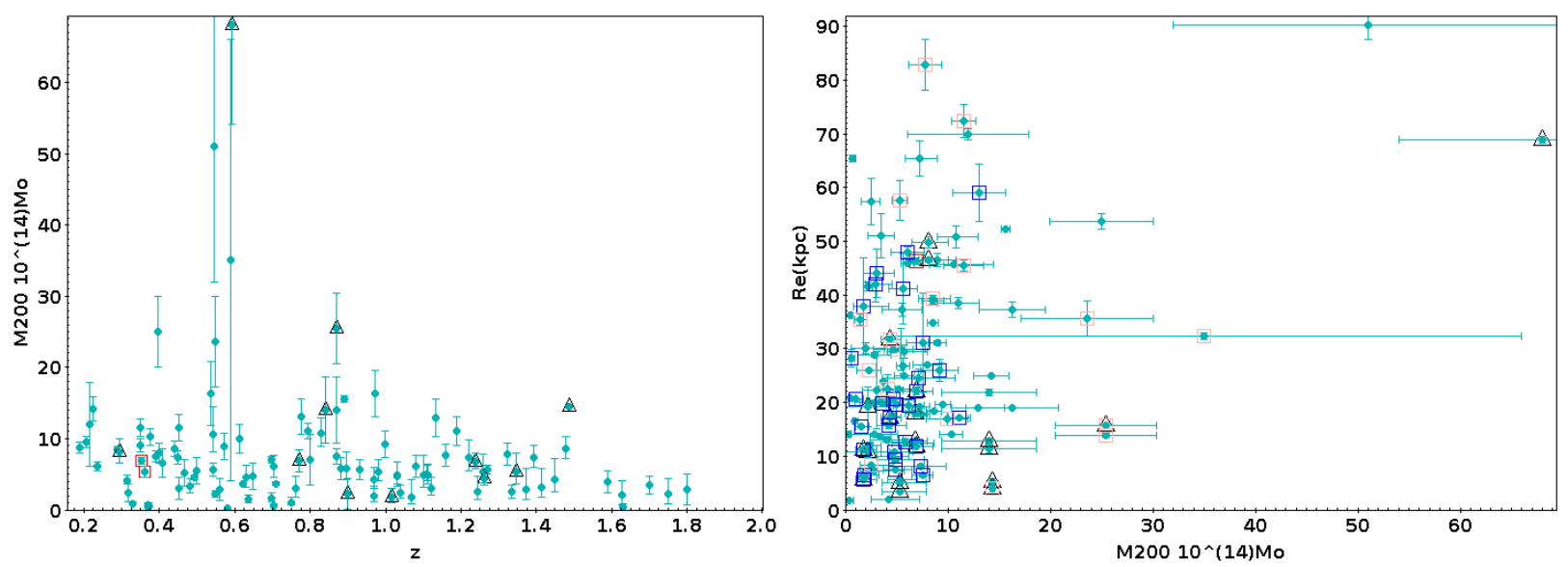

Fig. 20. Left: cluster mass M200 (in units of $10^{14} M_{\odot}$ ) as a function of redshift. Right: BCG effective radius as a function of cluster mass.
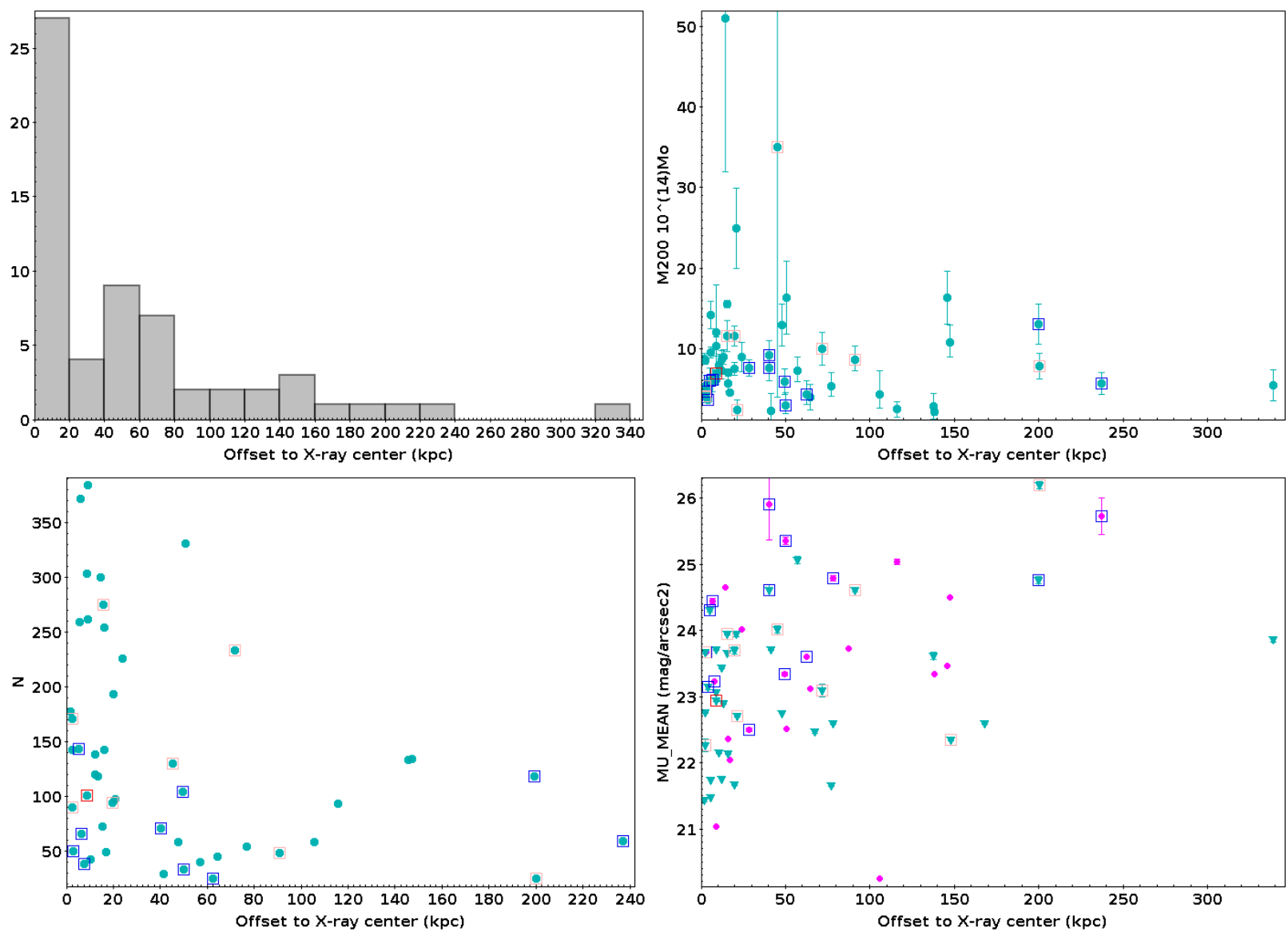

Fig. 21. Top left: histogram of the offsets between the BCG and the X-ray center. Top right: masses $M_{200, \mathrm{c}}$ of the clusters as a function of the offsets between the BCG and the cluster X-ray center. Bottom left: richness of the cluster as a function of the offsets between the BCG and the cluster $\mathrm{X}$-ray center. Bottom right: mean effective surface brightness of the BCGs as a function of the offsets between the BCG and the cluster X-ray center.

tighter alignment than that reported by Durret et al. (2019), who found an alignment for 12 out of 21 BCGs $(57 \%)$ between redshifts 0.21 and 0.89 . Okabe et al. (2020) study the alignment of 45 dark matter (DM) haloes and their BCGs up to $z=0.97$, and find that BCGs tend to be well aligned with their DM haloes, with a mean difference of $22.2 \pm 3.9^{\circ}$. A similar study was done by Ragone-Figueroa et al. (2020) on the alignment of BCGs both with the distribution of cluster galaxies and DM haloes, by analyzing cosmological hydro-simulations of 24 clusters. They find a strong alignment at $z<2$, and add that relaxed clusters tend to host BCGs that align with the major axis. Similar conclusions are made by De Propris et al. (2020), who show that BCGs are generally aligned with the host cluster even when the offset between the BCG and the X-ray center is significant.

Cerulo et al. (2019) found that $9 \%$ of the BCGs between $0.05 \leq z \leq 0.35$ have colors bluer than $2 \sigma$ of the median color of the cluster red sequence. During this study we found two peculiar blue BCGs in our sample. Apart from their colors and 
complex structures, these two peculiar BCGs do not have photometric properties different from the other BCGs. It would be interesting to continue this study by considering a larger sample of star forming blue BCGs to see where they lie in the previous plots.

We plan to apply the method described in this paper to more than a thousand clusters from the Canada-France-Hawaii Telescope Legacy Survey (CFHTLS), detected by Sarron et al. (2018), up to redshift $z=0.7$. This will enable us to better evaluate the accuracy of our BCG detection method on ground-based data, and although the resolution will not be as good, the sample will be significantly larger. We also found two BCGs (2\%) with blue colors, and it would be interesting to estimate the fraction of blue BCGs in the Universe up to redshift $z=0.7$. We wonder if these BCGs evolve differently from the red BCGs that we detect.

Acknowledgements. We thank the referee, M. West, for his constructive comments and suggestions. F.D. acknowledges continuous support from CNES since 2002. IM acknowledges financial support from the State Agency for Research of the Spanish MCIU through the "Center of Excellence Severo Ochoa" award to the Instituto de Astrofísica de Andalucía (SEV-2017-0709), and through the programs AYA2016-76682C3-1-P and PID2019-106027GB-C41. Based on observations made with the NASA/ESA Hubble Space Telescope, and obtained from the Hubble Legacy Archive, which is a collaboration between the Space Telescope Science Institute (STScI/NASA), the Space Telescope European Coordinating Facility (ST-ECF/ESA) and the Canadian Astronomy Data Centre (CADC/NRC/CSA).

\section{References}

Andreon, S., Newman, A. B., Trinchieri, G., et al. 2014, A\&A, 565, A120 Aragon-Salamanca, A., Baugh, C. M., \& Kauffmann, G. 1998, MNRAS, 297, 427

Ascaso, B., Aguerri, J. A. L., Varela, J., et al. 2010, ApJ, 726, 69

Bai, L., Yee, H., Yan, R., et al. 2014, ApJ, 789, 134

Bartalucci, I., Arnaud, M., Pratt, G. W., Démoclès, J., \& Lovisari, L. 2019, A\&A, 628, A86

Bellstedt, S., Lidman, C., Muzzin, A., et al. 2016, MNRAS, 460, 2862

Bernardi, M. 2009, MNRAS, 395, 1491

Bertin, E. 2011, in Astronomical Data Analysis Software and Systems XX, eds I. N. Evans, A. Accomazzi, D. J. Mink, \& A. H. Rots, ASP Conf. Ser., 442, 435

Bertin, E., \& Arnouts, S. 1996, A\&AS, 117, 393

Brodwin, M., Greer, C. H., Leitch, E. M., et al. 2015, ApJ, 806, 26

Bruzual, G., \& Charlot, S. 2003, MNRAS, 344, 1000

Castignani, G., Pandey-Pommier, M., Hamer, S. L., et al. 2020, A\&A, 640, A65

Cerulo, P., Orellana, G. A., \& Covone, G. 2019, MNRAS, 487, 3759

Chan, J. C. C., Wilson, G., Rudnick, G., et al. 2019, ApJ, 880, 119

Chiu, I., Mohr, J., McDonald, M., et al. 2015, MNRAS, 455, 258

Collins, C. A., \& Mann, R. G. 1998, MNRAS, 297, 128

Collins, C. A., Stott, J. P., Hilton, M., et al. 2009, Nature, 458, 603

Cooke, K. C., Kartaltepe, J. S., Tyler, K. D., et al. 2019a, ApJ, 881, 150

Cooke, E. A., Smail, I., Stach, S. M., et al. 2019b, MNRAS, 486, 3047

Czakon, N. G., Sayers, J., Mantz, A., et al. 2015, ApJ, 806, 18

Dahle, H. 2006, ApJ, 653, 954

De Lucia, G., \& Blaizot, J. 2007, MNRAS, 375, 2

De Propris, R., West, M. J., Andrade-Santos, F., et al. 2020, MNRAS, 500, 310

DeMaio, T., Gonzalez, A. H., Zabludoff, A., et al. 2019, MNRAS, 491, 3751

Donahue, M., Connor, T., Fogarty, K., et al. 2015, ApJ, 805, 177

Donahue, M., Ettori, S., Rasia, E., et al. 2016, ApJ, 819, 36

Donnarumma, A., Ettori, S., Meneghetti, M., \& Moscardini, L. 2009, MNRAS, 398,438

Durret, F., Márquez, I., Acebrón, A., et al. 2016, A\&A, 588, A69

Durret, F., Tarricq, Y., Márquez, I., Ashkar, H., \& Adami, C. 2019, A\&A, 622, A78

Edwards, L. O. V., Salinas, M., Stanley, S., et al. 2019, MNRAS, 491, 2617

Ellien, A., Durret, F., Adami, C., et al. 2019, A\&A, 628, A34

Fassbender, R., Böhringer, H., Nastasi, A., et al. 2011, New J. Phys., 13, 125014

Fogarty, K., Postman, M., Connor, T., Donahue, M., \& Moustakas, J. 2015, ApJ, 813,117

Fogarty, K., Postman, M., Larson, R., Donahue, M., \& Moustakas, J. 2017, ApJ, 846,103
Fogarty, K., Postman, M., Li, Y., et al. 2019, ApJ, 879, 103 Gonzalez, A. H., Decker, B., Brodwin, M., et al. 2015, ApJ, 812, L40 Green, T. S., Edge, A. C., Stott, J. P., et al. 2016, MNRAS, 461, 560 Guennou, L., Adami, C., Durret, F., et al. 2014, A\&A, 561, A112 Hashimoto, Y., Henry, J. P., \& Boehringer, H. 2014, MNRAS, 440, 588 Hennig, C., Mohr, J. J., Zenteno, A., et al. 2017, MNRAS, 467, 4015 Herbonnet, R., Sifón, C., Hoekstra, H., et al. 2020, MNRAS, 497, 4684 Hicks, A. K., Ellingson, E., Bautz, M., et al. 2008, ApJ, 680, 1022 Hicks, A. K., Pratt, G. W., Donahue, M., et al. 2013, MNRAS, 431, 2542 Hlavacek-Larrondo, J., Allen, S. W., Taylor, G. B., et al. 2013, ApJ, 777, 163 Hoekstra, H., Donahue, M., Conselice, C. J., McNamara, B. R., \& Voit, G. M. 2010, ApJ, 726, 48

Jee, M. J., White, R. L., Ford, H. C., et al. 2005, ApJ, 634, 813

Jee, M., Dawson, K., Hoekstra, H., et al. 2011, ApJ, 737, 59

Jee, M. J., Ko, J., Perlmutter, S., et al. 2017, ApJ, 847, 117

Jørgensen, I., Chiboucas, K., Hibon, P., Nielsen, L. D., \& Takamiya, M. 2018, ApJS, 235, 29

Just, D. W., Kirby, M., Zaritsky, D., et al. 2019, ApJ, 885, 6

Kluge, M., Neureiter, B., Riffeser, A., et al. 2020, ApJS, 247, 43

Kormendy, J. 1977, ApJ, 218, 333

Kravtsov, A. V., \& Borgani, S. 2012, ARA\&A, 50, 353

Lauer, T. R., Postman, M., Strauss, M. A., Graves, G. J., \& Chisari, N. E. 2014, ApJ, 797, 82

Lidman, C., Suherli, J., Muzzin, A., et al. 2012, MNRAS, 427, 550

Limousin, M., Richard, J., Jullo, E., et al. 2016, A\&A, 588, A99

Lin, Y.-T., Ostriker, J. P., \& Miller, C. J. 2010, ApJ, 715, 1486

Lin, Y.-T., Brodwin, M., Gonzalez, A. H., et al. 2013, ApJ, 771, 61

Margalef-Bentabol, B., Conselice, C. J., Mortlock, A., et al. 2016, MNRAS, 461, 2728

Márquez, I., Durret, F., Delgado, R. G., et al. 1999, A\&AS, 140, 1

Márquez, I., Masegosa, J., Durret, F., et al. 2003, A\&A, 409, 459

Martinet, N., Clowe, D., Durret, F., et al. 2016, A\&A, 590, A69

Martinet, N., Durret, F., Adami, C., \& Rudnick, G. 2017, A\&A, 604, A80

Martini, P., Miller, E. D., Brodwin, M., et al. 2013, ApJ, 768, 1

McDonald, M., Stalder, B., Bayliss, M., et al. 2016, ApJ, 817, 86

Merten, J., Meneghetti, M., Postman, M., et al. 2015, ApJ, 806, 4

Mo, W., Gonzalez, A., Jee, M. J., et al. 2016, ApJ, 818, L25

Noble, A. G., Webb, T. M. A., Ellingson, E., et al. 2011, MNRAS, 419, 1983

Okabe, N., \& Smith, G. P. 2016, MNRAS, 461, 3794

Okabe, T., Oguri, M., Peirani, S., et al. 2020, MNRAS, 496, 2591

Patel, P., Maddox, S., Pearce, F. R., Aragon-Salamanca, A., \& Conway, E. 2006, MNRAS, 370, 851

Peng, C. Y., Ho, L. C., Impey, C. D., \& Rix, H.-W. 2002, AJ, 124, 266

Pierpaoli, E., Borgani, S., Scott, D., \& White, M. 2003, MNRAS, 342, 163

Postman, M., Lauer, T. R., Donahue, M., et al. 2012a, ApJ, 756, 159

Postman, M., Coe, D., Benítez, N., et al. 2012b, ApJS, 199, 25

Ragone-Figueroa, C., Granato, G. L., Borgani, S., et al. 2020, MNRAS, 495, 2436

Richard-Laferrière, A., Hlavacek-Larrondo, J., Nemmen, R. S., et al. 2020, MNRAS, 499, 2934

Sanders, J. S., Fabian, A. C., Russell, H. R., \& Walker, S. A. 2017, MNRAS, 474, 1065

Santos, J. S., Tozzi, P., Rosati, P., Nonino, M., \& Giovannini, G. 2012, A\&A, 539, A105

Sarron, F., Martinet, N., Durret, F., \& Adami, C. 2018, A\&A, 613, A67

Sayers, J., Montaña, A., Mroczkowski, T., et al. 2019, ApJ, 880, 45

Sazonova, E., Alatalo, K., Lotz, J., et al. 2020, ApJ, 899, 85

Schlafly, E. F., \& Finkbeiner, D. P. 2011, ApJ, 737, 103

Schlegel, D., Finkbeiner, D., \& Davis, M. 1998, ApJ, 500, 525

Sereno, M. 2015, MNRAS, 450, 3665

Sereno, M., \& Covone, G. 2013, MNRAS, 434, 878

Sereno, M., \& Zitrin, A. 2011, MNRAS, 419, 3280

Simard, L., Mendel, J. T., Patton, D. R., Ellison, S. L., \& McConnachie, A. W. 2011, ApJS, 196, 11

Stott, J. P., Collins, C. A., Sahlén, M., et al. 2010, ApJ, 718, 23

Stott, J. P., Collins, C. A., Burke, C., Hamilton-Morris, V., \& Smith, G. P. 2011 , MNRAS, 414, 445

Thomas, D., Maraston, C., Schawinski, K., Sarzi, M., \& Silk, J. 2010, MNRAS, 404, 1775

Tozzi, P., Santos, J. S., Jee, M. J., et al. 2015, ApJ, 799, 93

van der Burg, R. F. J., Muzzin, A., Hoekstra, H., et al. 2014, A\&A, 561, A79

van der Burg, R. F. J., Muzzin, A., Hoekstra, H., et al. 2015, A\&A, 577, A19

West, M. J., de Propris, R., Bremer, M. N., \& Phillipps, S. 2017, Nat. Astron., 1, 0157

Whiley, I. M., Aragón-Salamanca, A., De Lucia, G., et al. 2008, MNRAS, 387, 1253

Zhang, Y., Miller, C., McKay, T., et al. 2016, ApJ, 816, 98

Zitrin, A., Meneghetti, M., Umetsu, K., et al. 2012, ApJ, 762, L30 